\title{
DeepTrack: Learning Discriminative Feature Representations Online for Robust Visual Tracking
}

\author{
Hanxi Li, Yi Li, Fatih Porikli
}

\begin{abstract}
Deep neural networks, albeit their great success on feature learning in various computer vision tasks, are usually considered as impractical for online visual tracking because they require very long training time and a large number of training samples. In this work, we present an efficient and very robust tracking algorithm using a single Convolutional Neural Network (CNN) for learning effective feature representations of the target object, in a purely online manner. Our contributions are multifold: First, we introduce a novel truncated structural loss function that maintains as many training samples as possible and reduces the risk of tracking error accumulation. Second, we enhance the ordinary Stochastic Gradient Descent approach in CNN training with a robust sample selection mechanism. The sampling mechanism randomly generates positive and negative samples from different temporal distributions, which are generated by taking the temporal relations and label noise into account. Finally, a lazy yet effective updating scheme is designed for CNN training. Equipped with this novel updating algorithm, the CNN model is robust to some long-existing difficulties in visual tracking such as occlusion or incorrect detections, without loss of the effective adaption for significant appearance changes. In the experiment, our CNN tracker outperforms all compared state-of-the-art methods on two recently proposed benchmarks which in total involve over 60 video sequences. The remarkable performance improvement over the existing trackers illustrates the superiority of the feature representations which are learned purely online via the proposed deep learning framework.
\end{abstract}

\section{INTRODUCTION}

Image features play a crucial role in many challenging computer vision tasks such as object recognition and detection. Unfortunately, in many online visual trackers features are manually defined and combined [1], [2], [3], [4]. Even though these methods report satisfactory results on individual datasets, hand-crafted feature representations would limit the performance of tracking. For instance, normalized cross correlation, which would be discriminative when the lighting condition is favourable, might become ineffective when the object moves under shadow. This necessitates good representation learning mechanisms for visual tracking that are capable of capturing the appearance effectively changes over time.

Recently, deep neural networks have gained significant attention thanks to their success on learning feature representations. Different from the traditional hand-crafted features [5], [6], [7], a multi-layer neural network architecture can efficiently capture sophisticated hierarchies describing the raw

$\mathrm{H}$. Li is with the School of Computer and Information Engineering, Jiangxi Normal University, Jiangxi, China. He is also with NICTA, Canberra Research Laboratory, Canberra, ACT2601, Australia, (e-mail: hanxi.li@nicta.com.au).

Y. Li and F. Porikli are with NICTA, Canberra Research Laboratory, Canberra, ACT2601, Australia, and also with the Research School of Information Science and Engineering, Australian National University, (e-mail: \{yi.li, fatih.porikli\}@nicta.com.au)

Correspondence should be addressed to Y. Li. data [8]. In particular, the Convolutional Neural Networks (CNN) has shown superior performance on standard object recognition tasks [9], [10], [11], [12], [13], which effectively learn complicated mappings while utilizing minimal domain knowledge.

However, the immediate adoption of CNN for online visual tracking is not straightforward. First of all, CNN requires a large number of training samples, which is often not be available in visual tracking as there exist only a few number of reliable positive instances extracted from the initial frames. Moreover, CNN tends to easily overfit to the most recent observation, e.g., most recent instance dominating the model, which may result in drift problem. Besides, CNN training is computationally intensive for online visual tracking. Due to these difficulties, CNN has been treated as an offline feature extraction step on predefined datasets [14], [15] for tracking applications so far.

In this work, we propose a novel tracking algorithm using CNN to automatically learn the most useful feature representations of particular target objects while overcoming the above challenges. We employ a tracking-by-detection strategy - a four-layer CNN model to distinguish the target object from its surrounding background. Our CNN generates scores for all possible hypotheses of the object locations (object states) in a given frame. The hypothesis with the highest score is then selected as the prediction of the object state in the current frame. We update this CNN model in an purely online manner. In other words, the proposed tracker is learned based only on the video frames for the interested object, no extra information or offline training is required.

Typically, tracking-by-detection approaches rely on predefined heuristics to sample from the estimated object location to construct a set of positive and negative samples. Often these samples have binary labels, which leads to a few positive samples and a large negative training set. However, it is well-known that CNN training without any pre-learned model usually requires a large number of training samples, both for positive ones and negative ones. Furthermore, even with sufficient samples, the learner usually needs hundreds of seconds to achieve a CNN model with an acceptable accuracy. The slow updating speed could prevent the CNN model from being a practical visual tracker. To address these two issues, our CNN model employs a special type of loss function that consists of a structural term and a truncated norm. The structural term makes it possible to obtain a large number of training samples that have different significance levels considering the uncertainty of the object location at the same time. The truncated norm is applied on the $\mathrm{CNN}$ response to reduce the number of samples in the back-propagation [9], 
[10] stage to significantly accelerate the training process.

We employ the Stochastic Gradient Decent (SGD) method to optimize the parameters in the CNN model. Since the standard SGD algorithm is not tailored for online visual tracking, we propose the following two modifications. First, to prevent the CNN model from overfitting to occasionally detected false positive instances, we introduce a temporal sampling mechanism to the batch generation in the SGD algorithm. This temporal sampling mechanism assumes that the object patches shall stay longer than those of the background in the memory. Therefore, we store all the observed image patches into training sample pool, and we choose the positive samples from a temporal range longer than the negative ones. In practice, we found this is a key factor in the robust CNN-based tracker, because discriminative sampling strategy successfully regularizes the training for effective appearance model. Secondly, the object locations, except the one on the first frame, is not always reliable as they are estimated by the visual tracker and the uncertainty is unavoidable [16]. One can treat this difficulty as the label noise problem [17], [18], [19]. We propose to sample the training data in the joint distribution over the temporal variable (frame index) and the sample class. Here we compute the conditional probability of the sample class, given the frame index, based on a novel measurement of the tracking quality in that frame. In the experiment, further performance improvement is observed when the sample class probability is taken into account.

For achieving a high generalization ability in various image conditions, we use multiple image cues (low-level image features, such as normalized gray-scale image and image gradient) as independent channels as network input. We update the CNN parameters by iteratively training each channel independently followed by a joint training on a fusion layer which replace the last fully-connected layers from multiple channels. The training processes of the independent channels and the fusion layer are totally decoupled. This makes the training efficient and empirically we observed that this twostage iterative procedure is more accurate than jointly training for all cues.

Finally, we propose to update the CNN model in a "lazy" style. First, the CNN-model is only updated when a significant appearance change occurs on the object. The intuition behind this lazy updating strategy is that we assume that the object appearance is more consistent over the video, compared with the background appearances. Second, the fusion layer is updated in a coordinate-descent style and with a lower learning rate. The underlying assumption is that the feature representations can be updated fast while the contribution ratios of different image cues are more stable over all the frames. In practice, this lazy updating strategy not only increases the tracking speed significantly but also yields observable accuracy increase.

To summarize, our main contributions include:

- A visual tracker based on online adapting CNN is proposed. As far as we are aware, this is the first time a single CNN is introduced for learning the best features for object tracking in an online manner.

- A structural and truncated loss function is exploited for the online CNN tracker. This enables us to achieve very reliable (best reported results in the literature) and robust tracking while achieving tracking speeds up to $4 \mathrm{fps}$.

- An iterative SGD method with an robust temporal sampling mechanism is introduced for competently capturing object appearance changes and meanwhile considering the label noise.

Our experiments on two recently proposed benchmarks involving over 60 videos demonstrate that our method outperforms all the compared state-of-the-art algorithms and rarely loses the track of the objects. In addition, it achieves a practical tracking speed (from $1.5 \mathrm{fps}$ to $4 \mathrm{fps}$ depending on the sequence and settings), which is comparable to many other visual trackers.

\section{CNN ARCHITECTURE}

\section{A. CNN with multiple image cues}

Our CNN consists of two convolutional layers and two fully-connected layers. The ReLU (Rectified Linear Unit) [10] is adopted as the activation function and max-pooling operators are used for dimension-reduction. The dark gray block in Fig. 1 shows the structure of our network, which can be expressed as $(32 \times 32) \rightarrow(10 \times 10 \times 12) \rightarrow(2 \times 2 \times 18) \rightarrow$ $(8) \rightarrow(2)$ in conventional neural network notation.

The input is locally normalized $32 \times 32$ image patches, which draws a balance between the representation power and computational load. The first convolution layer contains 12 kernels each of size $13 \times 13$ (an empirical trade-off between overfitting due to a very large number of kernels and discrimination power), followed by a pooling operation that reduces the obtained feature map (filter response) to a lower dimension. The second layer contains 216 kernels with size $7 \times 7$. This leads to a 72 -dimensional feature vector in the second convolutional layer, after the pooling operation in this layer.

The two fully connected layers firstly map the 72-D vector into a 8 -D vector and then generate a $2-\mathrm{D}$ confidence vector $\mathbf{s}=\left[s_{1}, s_{2}\right]^{\mathrm{T}} \in \mathcal{R}^{2}$, with $s_{1}$ and $s_{2}$ corresponding to the positive score and negative score, respectively. In order to increase the margin between the scores of the positive and negative samples, we calculate the $\mathrm{CNN}$ score of the patch $n$ as

$$
S\left(\mathbf{x}_{n} ; \Omega\right)=S_{n}=s_{1} \cdot \exp \left(s_{1}-s_{2}\right),
$$

where $\mathbf{x}_{n}$ denotes the input and the $\mathrm{CNN}$ is parameterized by the weights $\Omega$.

Effective object tracking requires multiple cues, which may include color, image gradients and different pixel-wise filter responses. These cues are weakly correlated yet contain complementary information. Local contrast normalized cues are previously shown [10] to produce accurate object detection and recognition results within the $\mathrm{CNN}$ frameworks. The normalization not only alleviates the saturation problem but also makes the $\mathrm{CNN}$ robust to illumination change, which is desired during the tracking. In this work, we use 3 image cues generated from the given gray-scale image, i.e., two locally 


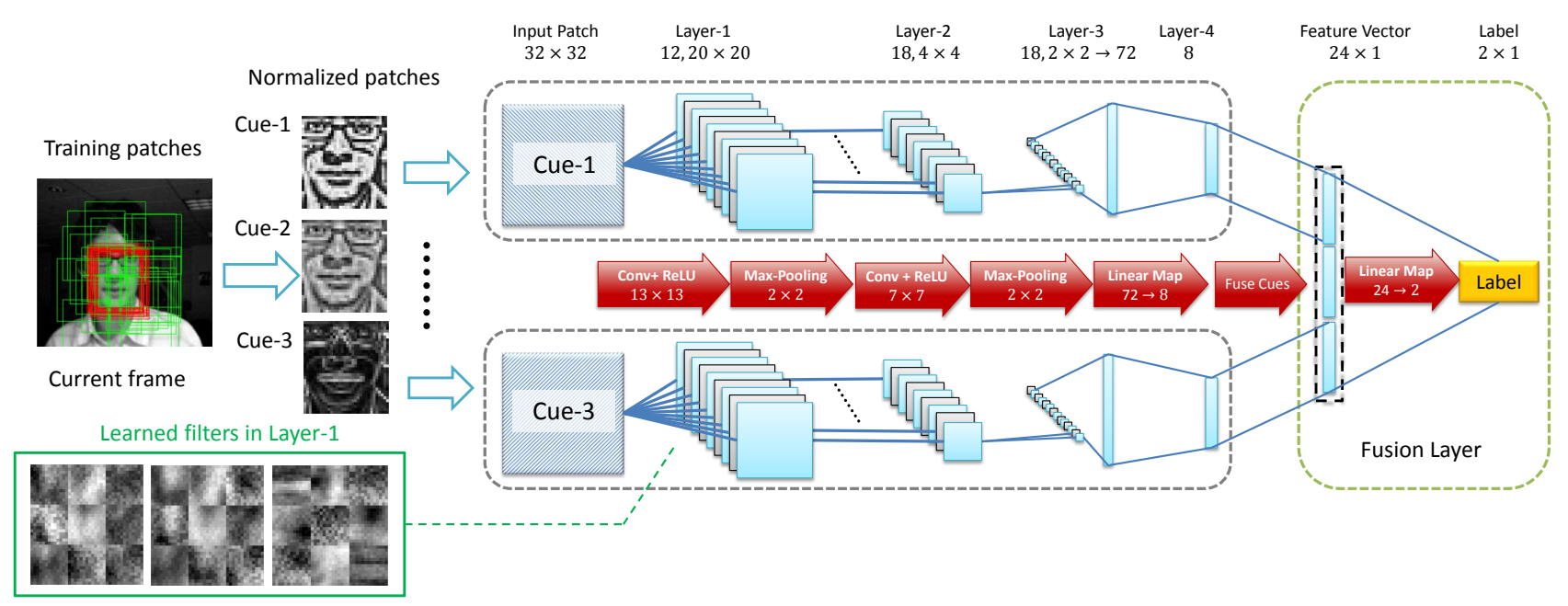

Fig. 1. The architecture of our CNN tracker with multiple image cues. The gray dashed blocks are the independent CNN channels for different image cues; the green dashed block is the fusion layer where a linear mapping $\mathbb{R}^{24} \rightarrow \mathbb{R}^{2}$ is learned.

normalized images with different parameter configurations 11 and a gradient image. For color images, the first two cues are simply replaced with the $\mathrm{H}$ and $\mathrm{V}$ channels of the HSV color representation. Offering multiple image cues, we then let CNN to select the most informative ones in a data driven fashion. By concatenating the final responses of these 3 cues, we build a fusion layer (the green dashed block in Fig. (1) to generate a 2-D output vector, based on which the final CNN score is calculated using Eq. 1 .

In our previous work [20], [21], we proposed to use a set of CNNs [20], or a single CNN [21] with multiple (4) image cues for visual tracking. In this work, we employ a more complex CNN model (as described above) while less image cues to strike the balance between robustness and tracking speed. Other small yet important modifications from the previous model includes:

- To better curb the overfitting, all the training samples are flipped as augmented data.

- The pixel values of each the image cue are normalized to the range $[0,10]$. We found this normalization is crucial for balancing the importances between different image cues.

\section{B. Structural and truncated loss function}

1) Structural loss: Let $\mathbf{x}_{n}$ and $\mathbf{l}_{n} \in\left\{[0,1]^{\mathrm{T}},[1,0]^{\mathrm{T}}\right\}$ denote the cue of the input patch and its ground truth label (background or foreground) respectively, and $f\left(\mathbf{x}_{n} ; \Omega\right)$ be the predicted score of $\mathbf{x}_{n}$ with network weights $\Omega$, the objective function of $N$ samples in the batch is

$$
\mathcal{L}=\frac{1}{N} \sum_{n=1}^{N}\left\|f\left(\mathbf{x}_{n} ; \Omega\right)-\mathbf{l}_{n}\right\|_{2}
$$

when the CNN is trained in the batch-mode. Eq. 2 is a commonly used loss function and performs well in binary

\footnotetext{
${ }^{1}$ Two parameters $r_{\mu}$ and $r_{\sigma}$ determine a local contrast normalization process. In this work, we use two configurations, i.e., $\left\{r_{\mu}=8, r_{\sigma}=8\right\}$ and $\left\{r_{\mu}=12, r_{\sigma}=12\right\}$, respectively.
}

classification problems. However, for object localization tasks, usually higher performance can be obtained by 'structurizing' the binary classifier. The advantage of employing the structural loss is the larger number of available training samples, which is crucial to the $\mathrm{CNN}$ training. In the ordinary binaryclassification setting, one can only use the training samples with high confidences to avoid class ambiguity. In contrast, the structural CNN is learned based upon all the sampled patches.

We modify the original CNN's output to $f\left(\phi\left\langle\Gamma, \mathbf{y}_{n}\right\rangle ; \Omega\right) \in$ $\mathbb{R}^{2}$, where $\Gamma$ is the current frame, $\mathbf{y}_{n} \in \mathbb{R}^{o}$ is the motion parameter vector of the target object, which determines the object's location in $\Gamma$ and $o$ is the freedom degree $2^{2}$ of the transformation. The operation $\phi\left\langle\Gamma, \mathbf{y}_{n}\right\rangle$ suffices to crop the features from $\Gamma$ using the motion $\mathbf{y}_{n}$. The associated structural loss is defined as

$$
\mathcal{L}=\frac{1}{N} \sum_{n=1}^{N}\left[\Delta\left(\mathbf{y}_{n}, \mathbf{y}^{*}\right) \cdot\left\|f\left(\phi\left\langle\Gamma, \mathbf{y}_{n}\right\rangle ; \Omega\right)-\mathbf{l}_{n}\right\|_{2}\right],
$$

where $\mathbf{y}^{*}$ is the (estimated) motion state of the target object in the current frame. To define $\Delta\left(\mathbf{y}_{n}, \mathbf{y}^{*}\right)$ we first calculate the overlapping score $\Theta\left(\mathbf{y}_{n}, \mathbf{y}^{*}\right)[22]$ as

$$
\Theta\left(\mathbf{y}_{n}, \mathbf{y}^{*}\right)=\frac{\operatorname{area}\left(r\left(\mathbf{y}_{n}\right) \bigcap r\left(\mathbf{y}^{*}\right)\right)}{\operatorname{area}\left(r\left(\mathbf{y}_{n}\right) \bigcup r\left(\mathbf{y}^{*}\right)\right)}
$$

where $r(\mathbf{y})$ is the region defined by $\mathbf{y}, \bigcap$ and $\bigcup$ denotes the intersection and union operations respectively. Finally we have

$$
\Delta\left(\mathbf{y}_{n}, \mathbf{y}^{*}\right)=\left|\frac{2}{1+\exp \left(-\left(\Theta\left(\mathbf{y}_{n}, \mathbf{y}^{*}\right)-0.5\right)\right)}-1\right| \in[0,1] .
$$

And the sample label $\mathbf{l}_{n}$ is set as.

$$
\mathbf{l}_{n}= \begin{cases}{[1,0]^{\mathrm{T}}} & \text { if } \Theta\left(\mathbf{y}_{n}, \mathbf{y}^{*}\right)>0.5 \\ {[0,1]^{\mathrm{T}}} & \text { elsewise }\end{cases}
$$

From Eq. 5 we can see that $\Delta\left(\mathbf{y}_{n}, \mathbf{y}^{*}\right)$ actually measures the importance of the training patch $n$. For instance, patches that

\footnotetext{
${ }^{2}$ In this paper $o=3$, i.e., the bounding box changes in its location and the scale.
} 
are very close to object center and reasonably far from it may play more significant roles in training the $\mathrm{CNN}$, while the patches in between are less important.

In visual tracking, when a new frame $\Gamma_{(t)}$ comes, we predict the object motion state $\mathbf{y}_{(t)}^{*}$ as

$$
\mathbf{y}_{(t)}^{*}=\underset{\mathbf{y}_{n} \in \mathcal{Y}}{\arg \max }\left(f\left(\phi\left\langle\Gamma_{(t)}, \mathbf{y}_{n}\right\rangle ; \Omega\right)\right),
$$

where $\mathcal{Y}$ contains all the test patches in the current frame.

2) Truncated structural loss: Ordinary CNN models regress the input features into the target labels, via the $l_{2}$-norm loss. One can directly adopt this strategy in the CNN-based tracking algorithm. However, to speed up the online training process, we employ a truncated $l_{2}$-norm in our model. We empirically observe that patches with very small error does not contribute much in the back propagation. Therefore, we can approximate the loss by counting the patches with errors that are larger than a threshold. Motived by this, in [21], we define a truncated $l_{2}$ norm as

$$
\|e\|_{\mathbb{T}}=\|e\|_{2} \cdot\left(1-\mathbb{1}\left[\|e\|_{2} \leq \beta\right]\right),
$$

where $\mathbb{1}[\cdot]$ denotes the indicator function while $e$ is the prediction error, e.g., $f\left(\phi\left\langle\Gamma, \mathbf{y}_{n}\right\rangle ; \Omega\right)-\mathbf{l}_{n}$ for patch- $n$. In our previous work [21], this truncated loss did increase the training speed, while at the cost of reducing the prediction accuracy.

In this work, we observed that the tracking performance is more sensitive to the prediction error on positive samples than the negative samples. Recall that in training stage, we label each positive sample as $[1,0]^{T}$ and each negative sample as $[0,1]^{T}$. In the test stage, the visual tracker selects the best particle among the ones with high scores. If the highest score in the current frame is large enough, the negative samples with small errors, which are ignored in training according to the truncated loss, will not affect the prediction. In contrast, if one ignores the positive samples with small errors in training, the selection among the top- $n$ particles in the test stage will be consequently inaccurate, and thus drift problems could happen. In other words, we need a more precise loss function for positive samples in visual tracking. We thus improve the original truncated loss function as:

$$
\|e\|_{\mathbb{T}}=\|e\|_{2} \cdot\left(1-\mathbb{1}\left[\|e\|_{2} \leq \frac{\beta}{\left(1+u \cdot l_{n}\right)}\right]\right)
$$

where $u>0$ and $l_{n}=\mathbf{l}_{n}(1)$, i.e., the scalar label of the $n$-th sample. This truncated norm is visualized in Fig. 2 and now Eq. 3 becomes:

$$
\mathcal{L}=\frac{1}{N} \sum_{n=1}^{N}\left[\Delta\left(\mathbf{y}_{n}, \mathbf{y}^{*}\right) \cdot\left\|f\left(\phi\left\langle\Gamma, \mathbf{y}_{n}\right\rangle ; \Omega\right)-\mathbf{l}_{n}\right\|_{\mathbb{T}}\right],
$$

It is easy to see that with the truncated norm $\|\cdot\|_{\mathbb{T}}$, the backpropagation [9] process only depends on the training samples with large errors, i.e., $\left\|f\left(\phi\left\langle\Gamma, \mathbf{y}_{n}\right\rangle ; \Omega\right)-\mathbf{l}_{n}\right\|_{\mathbb{T}}>0$. Accordingly, we can ignore the samples with small errors and the backpropagation procedure is significantly accelerated. In this work, we use $\beta=0.0025$ and $u=3$.

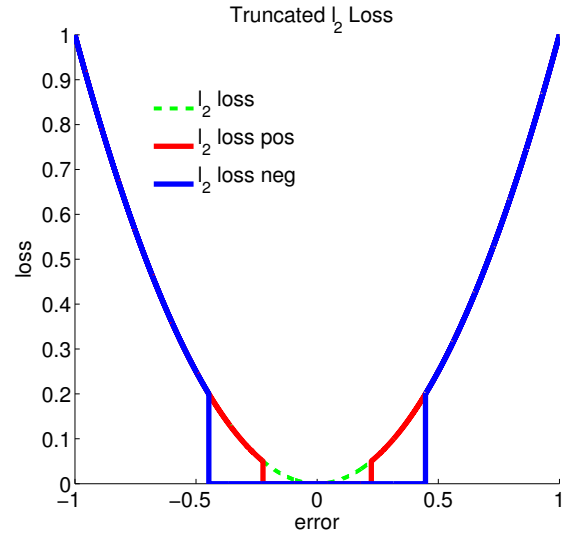

Fig. 2. The truncated $l_{2}$ losses. The dashed green curve indicates the original $l_{2}$ loss, the red and blue curves are the truncated losses for positive and negative samples.

\section{OPTIMIZATION OF CNN FOR TRACKING}

\section{A. Online Learning: Iterative SGD with Temporal Sampling}

1) Temporal Sampling: Following other CNN-based approaches [9], [10], we used Stochastic Gradient Decent (SGD) for the learning of the parameters $\Omega$. However, the SGD we employ is specifically tailored for visual tracking.

Different from detection and recognition tasks, the training sample pool grows gradually as new frames come in visual tracking. Moreover, it is desired to learn a consistent object model over all the previous frames and then use it to distinguish the object from the background in the current frame. This implies that we can effectively learn a discriminative model on a long-term positive set and a short-term negative set.

Based on this intuition, we tailor the SGD method by embedding in a temporal sampling process. In particular, given that the positive sample pool is $\mathbb{Y}_{1: t}^{+}=$ $\left.\left\{\mathbf{y}_{1,(1)}^{+}, \mathbf{y}_{2,(1)}^{+}, \ldots, \mathbf{y}_{N-1,(t)}^{+}, \mathbf{y}_{N,(t)}^{+}\right\}\right\}^{3}$ and the negative sample pool is $\mathbb{Y}_{1: t}^{-}=\left\{\mathbf{y}_{1,(1)}^{-}, \mathbf{y}_{2,(1)}^{-}, \ldots, \mathbf{y}_{N-1,(t)}^{-}, \mathbf{y}_{N,(t)}^{-}\right\}$, when generating a mini-batch for SGD, we sample the positive pool with the probability

$$
\operatorname{Prob}\left(\mathbf{y}_{n,\left(t^{\prime}\right)}^{+}\right)=\frac{1}{t N}
$$

while sample the negative samples with the probability

$$
\operatorname{Prob}\left(\mathbf{y}_{n,\left(t^{\prime}\right)}^{-}\right)=\frac{1}{Z} \exp \left[-\sigma\left(t-t^{\prime}\right)^{2}\right],
$$

where $\frac{1}{Z}$ is the normalization term and we use $\sigma=10$ in this work.

In a way, the above temporal selection mechanism can be considered to be similar to the "multiple-lifespan" data sampling [23]. However, [23] builds three different codebooks, each corresponding to a different lifespan, while we learn one discriminative model based on two different sampling distributions.

\footnotetext{
${ }^{3}$ Here we slightly abuse the notation of $\mathbf{y}$, which denotes the motion state in the previous section. Here $\mathbf{y}$ indicates the cropped image patch according to the motion state.
} 
2) Robust Temporal Sampling with Label Noise: In most tracking-by-detection strategy, the detected object $\mathbf{y}_{(t)}^{*}$ is treated as a true-positive in the following training stage. However, among all the motion states $\mathbf{y}_{(t)}^{*}, \forall t=1,2, \ldots, T$, only the first one $\mathbf{y}_{(1)}^{*}$ is always reliable as it is manually defined. Other motion states are estimated based on the previous observations. Thus, the uncertainty of the prediction $\mathbf{y}_{(t)}, \forall t>1$ is usually unavoidable [16]. Recall that, the structural loss defined in Eq. 4 could change significantly if a minor perturbation is imposed on $\mathbf{y}_{(t)}$, one requires a accurate $\mathbf{y}_{(t)}$ in every frame, which is, unfortunately, not feasible.

In our previous work [21], we take the uncertainty into account by imposing a robust term on the loss function 9 . The robust term is designed in the principle of Multiple-InstanceLearning [24], [25] and it alleviates over-fittings in some scenarios. However, the positive-sample-bag [21] could also reduce the learning effectiveness as it will confuse the learner when two distinct samples are involved in one bag. Actually, other MIL-based trackers also suffer from this problem [16], [24].

In this work, we propose a much simpler scheme for addressing the issue of prediction uncertainty. Specifically, the prediction uncertainty is casted as a label noise problem [17], [18], [19]. We assume there exist some frames, on which the detected "objects" are false-positive samples. In other words, the some sample labels in $\mathbb{Y}_{1: t}^{+}$and $\mathbb{Y}_{1: t}^{-}$are contaminated (flipped in the binary case). In the context of temporal sampling, the assumption introduces an extra random variable $\boldsymbol{\eta}$ which represent the event that the label is true $(\boldsymbol{\eta}=1)$ or not $(\boldsymbol{\eta}=0)$. The sampling process is now conduct in the joint probability space $\{n=1,2, \cdots, N\} \times\left\{t^{\prime}=\right.$ $1,2, \cdots, t\} \times\{\boldsymbol{\eta}=1,0\}$ and the joint probability is

$$
\operatorname{Prob}\left(\mathbf{y}_{n,\left(t^{\prime}\right)}^{ \pm}, \boldsymbol{\eta}=1\right),
$$

where $\mathbf{y}_{n,\left(t^{\prime}\right)}^{ \pm}$stands for the selection of the $n$-th positive/negative sample in the $t^{\prime}$-th frame. According to the chainrule, we have

$$
\begin{aligned}
\operatorname{Prob}\left(\mathbf{y}_{n,\left(t^{\prime}\right)}^{ \pm}, \boldsymbol{\eta}=1\right)=\operatorname{Prob}\left(t^{\prime}, n, \boldsymbol{\eta}=1\right) \\
=\operatorname{Prob}\left(\boldsymbol{\eta}=1 \mid t^{\prime}, n\right) \cdot \operatorname{Prob}\left(t^{\prime}, n\right) \\
=\operatorname{Prob}\left(\boldsymbol{\eta}=1 \mid t^{\prime}, n\right) \cdot \operatorname{Prob}\left(\mathbf{y}_{n,\left(t^{\prime}\right)}^{ \pm}\right)
\end{aligned}
$$

where $\operatorname{Prob}\left(\mathrm{y}_{n,\left(t^{\prime}\right)}^{ \pm}\right)$is given in Eq. 10 and 11 while the conditional probability $\operatorname{Prob}\left(\boldsymbol{\eta}=1 \uparrow t^{\prime}, n\right)$ reflects the likelihood that the label of sample $\mathbf{y}_{n,\left(t^{\prime}\right)}^{ \pm}$is not contaminated.

To estimate $\operatorname{Prob}\left(\boldsymbol{\eta}=1 \mid t^{\prime}, n\right)$ efficiently, we assume that in the same frame, the conditional probabilities are equal for $\forall n=1,2, \cdots, N$. Then we propose to calculate the probability as a prediction quality $\mathrm{Q}_{t^{\prime}}$ in frame- $t^{\prime}$, i.e.,

$$
\begin{aligned}
& \mathrm{Q}_{t^{\prime}}=\operatorname{Prob}\left(\boldsymbol{\eta}=1 \mid t^{\prime}, n\right)= \\
& 1-\frac{1}{|\mathbb{P}|} \sum_{n \in \mathbb{P}}^{N}\left[\Delta\left(\mathbf{y}_{n,\left(t^{\prime}\right)}, \mathbf{y}_{\left(t^{\prime}\right)}^{*}\right) \cdot\left\|f\left(\phi\left\langle\Gamma, \mathbf{y}_{n,\left(t^{\prime}\right)}\right\rangle ; \Omega\right)-\mathbf{l}_{n}\right\|_{\mathbb{T}}\right],
\end{aligned}
$$

where the set $\mathbb{P}$ contains the sample in the frame $t^{\prime}$ with high scores. Mathematically, it is defined as

$$
\mathbb{P}=\left\{\forall n \mid S_{n,\left(t^{\prime}\right)}>v \cdot S_{\left(t^{\prime}\right)}^{*}\right\},
$$

where $S_{n,\left(t^{\prime}\right)}$ and $S_{\left(t^{\prime}\right)}^{*}$ are the CNN scores (see Eq. 1p of the $n$-th sample and the sample selected as object in frame $t^{\prime}$, respectively. The underlying assumption of Eq. 14 is that, a detection heat-map with multiple widely-distributed peaks usually implies low detection quality, as there is only ONE target in the video sequence. This tracking quality is illustrated in Fig. 3. From the figure we can see that when occlusion (middle) or significant appearance change (right) occurs, the tracking quality drops dramatically and thus the samples in those "contaminated" frames are rarely selected according to Eq. 13 .

3) Iterative Stochastic Gradient Descent (IT-SGD): Recall that we use multiple image cues as the input of the CNN tracker. This leads to a CNN with higher complexity, which implies a low training speed and a high possibility of overfitting. By noticing that each image cue may be weakly independent, we train the network in a iterative manner. In particular, we define the model parameters as $\Omega=\left\{\mathbf{w}_{\text {cov }}^{1}, \cdots, \mathbf{w}_{\text {cov }}^{K}, \mathbf{w}_{f c}^{1}, \cdots, \mathbf{w}_{f c}^{K}, \mathbf{w}_{\text {fuse }}\right\}$, where $\mathbf{w}_{\text {cov }}^{k}$ denotes the filter parameters in cue- $k, \mathbf{w}_{f c}^{k}$ corresponds to the fully-connected layers and $\mathbf{w}_{\text {fuse }}$ parameterize the fusion layer.

In this work, we conduct the SGD process iteratively over different image cues and the fusion layer. In specific, after we complete the training on $\mathbf{w}_{c o v}^{k}$ and $\mathbf{w}_{f c}^{k}$, we evaluate the filter responses from that cue in the last fully-connected layer and then update $\mathbf{w}_{\text {fuse }}$ on the dimensions corresponding to cue- $k$. This can be regarded as a coordinate-descent variation of SGD. In practice, we found out both the robust temporal sampling mechanism and the IT-SGD significantly curb the overfitting problem. The iterative SGD is illustrated in Algorithm 1.

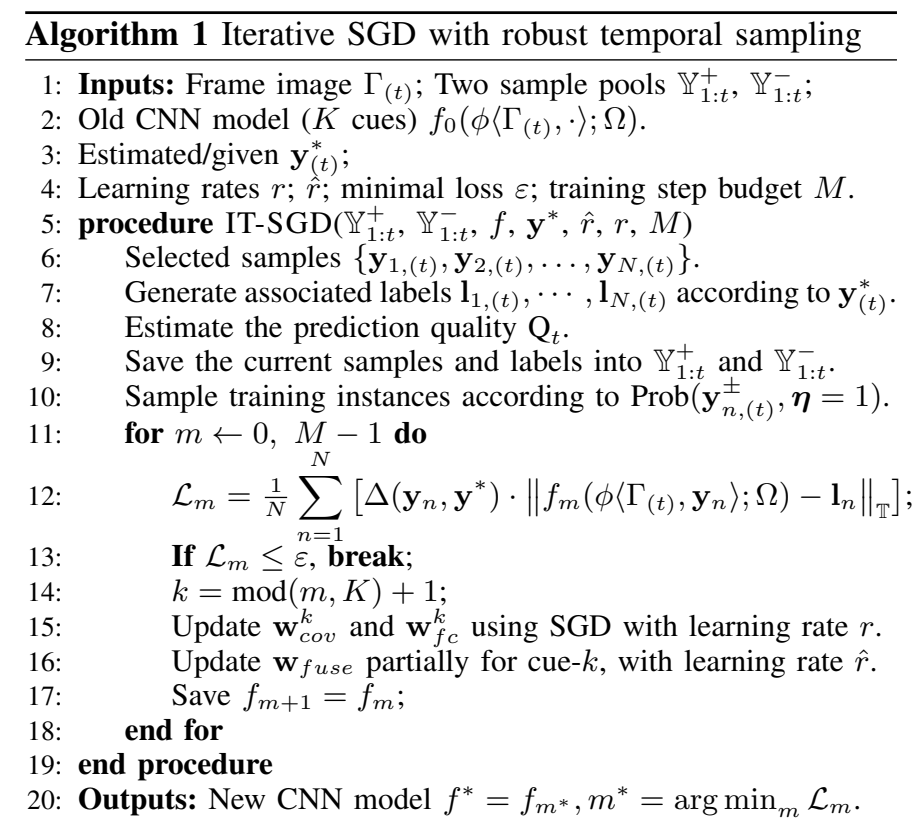



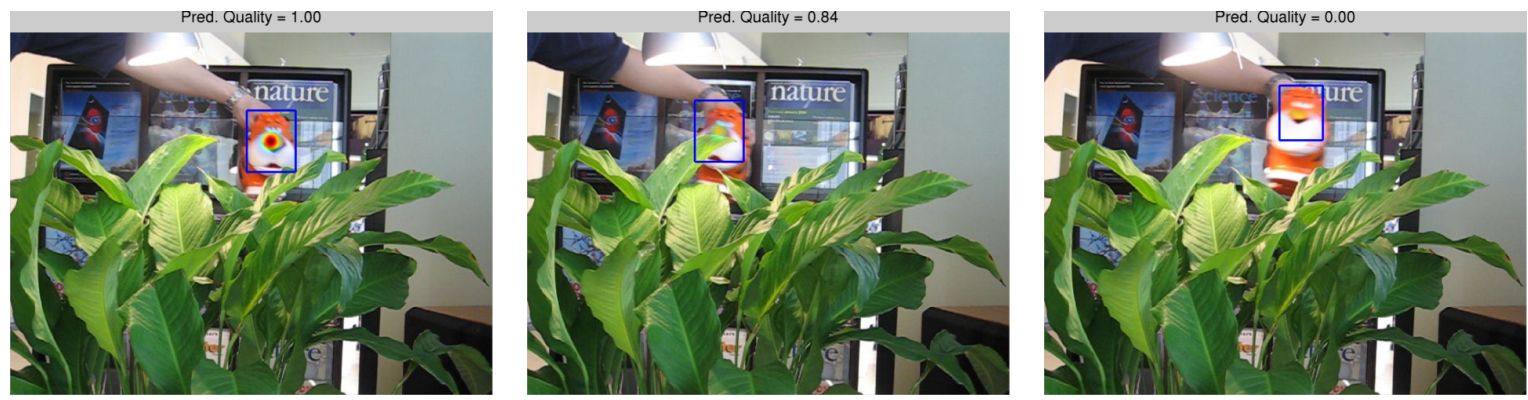

Fig. 3. A demonstration of the prediction quality on three different frames in the sequence tiger1. For each frame, the overlaying heat-map indicates the distribution of the high-score particles while the blue box is the detected $\mathbf{y}_{\left(t^{\prime}\right)}^{*}$. The tracking qualities are shown on the top of the frame images. Note that the quality is estimated without ground-truth information.

\section{B. Lazy Update and the Overall Work Flow}

It is straightforward to update the CNN model using the IT-SGD algorithm at each frame. However, this could be computationally expensive as the complexity of training processes would dominate the complexity of the whole algorithm. On the other hand, in case the appearance of the object is not always changing, a well-learned appearance model can remain discriminant for a long time. Furthermore, when the feature representations is updated for adapting the appearance changes, the contribution ratios of different image cues could remain more stable over all the frames.

Motivated by the above two intuitions, we propose to update the CNN model in a lazy manner. First, when tracking the object, we only update the $\mathrm{CNN}$ model when the training loss $\mathcal{L}_{1}$ is above $2 \varepsilon$. Once the training start, the training goal is to reduce $\mathcal{L}$ below $\varepsilon$. As a result, usually $\mathcal{L}_{1}<2 \varepsilon$ holds in a number of the following frames, and thus no training is required for those frames. This way, we accelerate the tracking algorithm significantly (Fig. 4). Second, we update the fusion layer in a lazy, i.e., a coordinate-descent manner with a small learning rate (see Algorithm 11). The learning process is thus stabilized well. In this work, we set that $\varepsilon=5 \mathrm{e}-3, r=5 \mathrm{e}-2$ and $\hat{r}=5 \mathrm{e}-3$.

\section{EXPERIMENTS}

\section{A. Benchmarks and experiment setting}

We evaluate our method on two recently proposed visual tracking benchmarks, i.e., the CVPR2013 Visual Tracker Benchmark [26] and the VOT2013 Challenge Benchmark [27]. These two benchmarks contain more than 60 sequences and cover almost all the challenging scenarios such as scale changes, illumination changes, occlusions, cluttered backgrounds and motion blur. Furthermore, these two benchmarks evaluate tracking algorithms with different measures and criteria, which can be used to analyze the tracker from different views.

In the experiments on two selected benchmarks, we use the same parameter values for DeepTrack. Most parameters of the $\mathrm{CNN}$ tracker are given in Sec. II and Sec. III In addition, there are some motion parameters for sampling the image patches. In this work, we only consider the displacement $\Delta_{x}, \Delta_{y}$ and the relative scale $s$ of the object $\left.\right|^{4}$. In a new frame, we sample 1500 random patches in a Gaussian Distribution which centers on the previous predicted state. The standard deviation for the three dimensions are $\min (10,0.5 \cdot h), \min (10,0.5 \cdot h)$ and 0.01 . $h$, respectively. Note that, all parameters are fixed for all videos in both two benchmarks; no parameter tuning is performed for any specific video sequence. We run our algorithm in Matlab with an unoptimized code mixed with CUDA-PTX kernels for the CNN implementation. The hardware environment includes one quad-core CPU and a NVIDIA GTX980 GPU.

\section{B. Comparison results on the CVPR2013 benchmark}

The CVPR2013 Visual Tracker Benchmark [26] contains 50 fully annotated sequences. These sequences include many popular sequences used in the online tracking literature over the past several years. For better evaluation and analysis of the strength and weakness of tracking approaches, these sequences are annotated with the 11 attributes including illumination variation, scale variation, occlusion, deformation, motion blur, fast motion, in-plane rotation, out-of-plane rotation, out-ofview, background clutters, and low resolution. The benchmark contains the results of 29 tracking algorithms published before the year 2013. Here, we compare our method with other 11 tracking methods. Among the competitors, TPGR [28] and KCF [29] are the most recently state-of-the-art visual trackers; TLD [30], VTD [31], CXT [32], ASLA [33], Struck [4], SCM [34] are the top- 6 methods as reported in the benchmark; CPF [1], IVT [35] and MIL [16] are classical tracking methods which are used as comparison baselines.

The tracking results are evaluated via the following two measurements: 1) Tracking Precision (TP), the percentage of the frames whose estimated location is within the given distance-threshold $\left(\tau_{d}\right)$ to the ground truth, and 2) Tracking Success Rate (TSR), the percentage of the frames in which the overlapping score defined in Eq. 4 between the estimated location and the ground truth is larger than a given overlapping-threshold $\left(\tau_{o}\right)$. Following the setting in the recently published work [28], [29], we conduct the experiment using the OPE (one-pass evaluation) evaluation strategy for a better comparison to the latest methods.

\footnotetext{
${ }^{4} s=h / 32$, where $h$ is object's height
} 


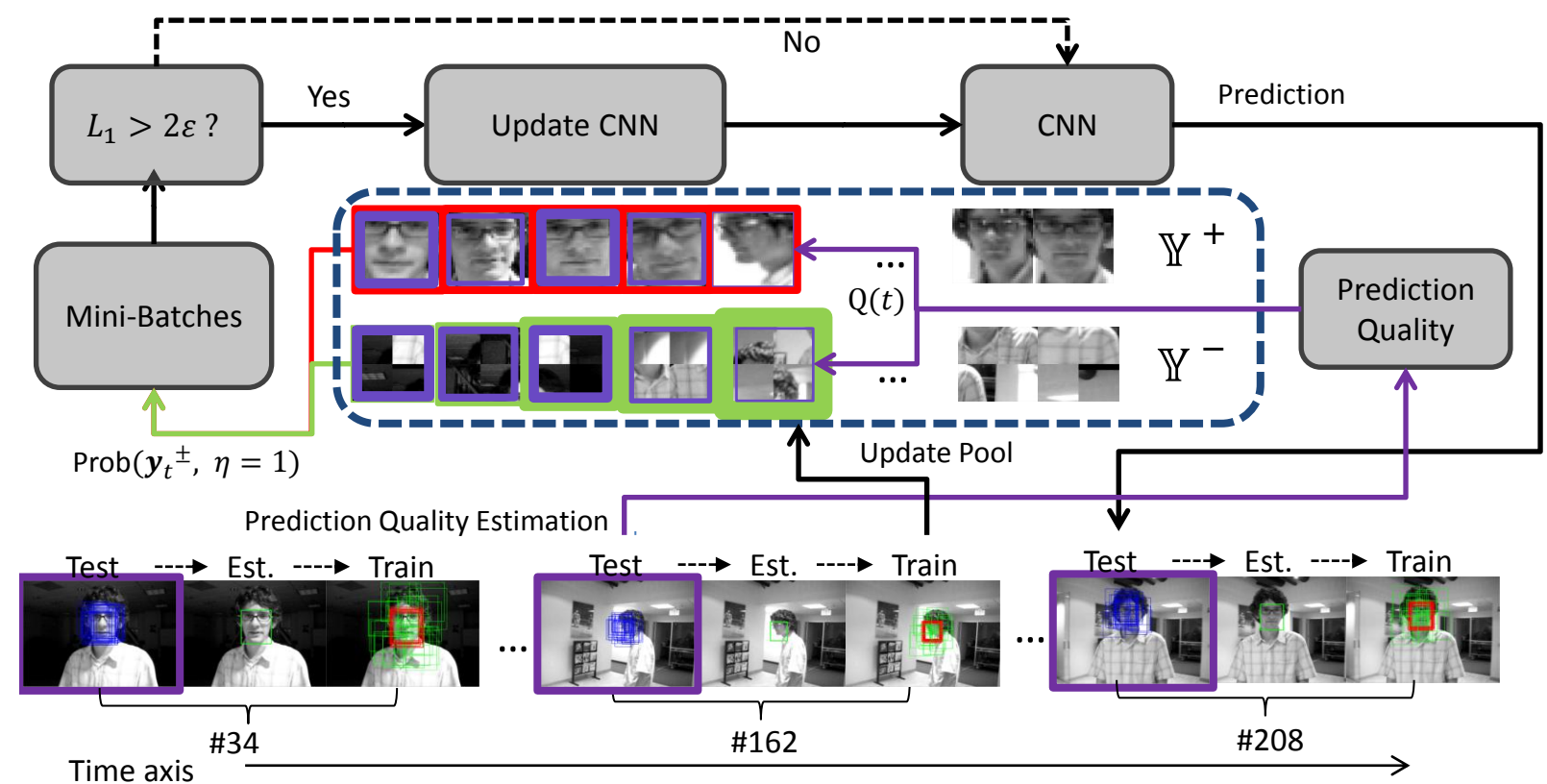

Fig. 4. Work flow of proposed algorithm. The bottom row shows the three-stages operations on a frame: test, estimation and training. In the training frames, the green bounding-boxes are the negative samples while the red ones denote the positive samples. The dashed block covers the positive sample pool $\mathbb{Y}^{+}$ (red) and negative sample pool $\mathbb{Y}^{-}$(green). In each pool, the edges of the sample patches indicate their sampling importances. The green ones (negative) and red ones (positive) represent the prior probabilities of sample selection while the purple ones stands for the conditional probabilities $(\mathrm{Q}(t))$. The thicker the edge, the higher the probability.

Firstly, we evaluate all algorithms using fixed thresholds, i.e., $\tau_{d}=20, \tau_{o}=0.6$, which is a standard setting in tracking evaluations [26]. Results for all the involved trackers and all the video sequences are given in Table 1 . According to the table, our method achieves better average performance compared with other trackers. The performance gap between our method and the reported best result in the literature are $6 \%$ for the TP measure: our method achieves $83 \%$ accuracy while the best state-of-the-art is 77\% (TGPR method). For the TSR measure, our method is $8 \%$ better than the existing methods: our method gives $63 \%$ accuracy while the best state-of-theart is 55\% (SCM method). Furthermore, our CNN tracker have ranked as the best method for 33 times. These numbers for TGPR, KCF, SCM and Struck are 21, 28, 19 and 21

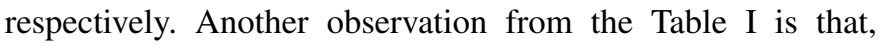
DeepTrack rarely performs inaccurately; there are only 36 occasions when the proposed tracker performs significantly poorer than the best method (no less then $80 \%$ of the highest score for one sequence).

In fact, the superiority of our method becomes more clear when the tracking result are evaluated using different measurement criteria (different $\tau_{d}, \tau_{o}$ ). In specific, for TP, we evaluate the trackers with the thresholds $\tau_{d}=1,2, \cdots, 50$ while for TSR, we use the thresholds $\tau_{o}=0$ to 1 at the step of 0.05 . Accordingly we generate the precision curves and the successrate curves for each tracking method, which is shown in Fig. 5.

From the score plots we can see that, overall the CNN tracker ranks the first (red curves) for both TP and TSR evaluations. The proposed DeepTrack method outperform all the other trackers when $\tau_{o}<0.68$ and $\tau_{d}>10$. When the evaluation threshold is reasonably loose, (i.e., $\tau_{o}<0.45$ and $\tau_{d}>20$ ), our algorithm is very robust with both the accuracies higher than $80 \%$. Having mentioned that when the overlap thresholds are tight (e.g. $\tau_{o}>0.75$ or $\left.\tau_{d}<5\right)$, our tracker has similar response to rest of the trackers we tested.

In many applications, it is more important to not to loose the target object than very accurately locate its bounding box. As visible, our tracker rarely looses the object. It achieves the accuracies around $90 \%$ when $\tau_{o}<0.3$ and $\tau_{d}>30$.

Fig. 6 shows the performance plots for 11 kinds of difficulties in visual tracking, i.e., fast-motion, background-clutter, motion-blur, deformation, illumination-variation, in-planerotation, low-resolution, occlusion, out-of-plane-rotation, outof-view and scale-variations. We can see that the proposed DeepTrack outperforms other competitors for all the difficulties except the "out-of-view" category.

\section{Comparison results on the VOT2013 benchmark}

The VOT2013 Challenge Benchmark [27] provides an evaluation kit and the dataset with 16 fully annotated sequences for evaluating tracking algorithms in realistic scenes subject to various common conditions. The tracking performance in the VOT2013 Challenge Benchmark is primarily evaluated with two evaluation criteria: accuracy and robustness. The accuracy measure is the average of the overlap ratios over the valid frames of each sequence while the tracking robustness is the average number of failures over 15 runs. A tracking failure happens once the overlap ratio measure drops to zero and an re-initialization of the tracker in the failure frame is conducted so it can continue. According to the evaluation protocol, three types of experiments are conducted. In Experiment-1, the tracker is run on each sequence in the dataset 15 times by 


\begin{tabular}{|c|c|c|c|c|c|c|c|c|c|c|c|c|}
\hline & Struck & MIL & VTD & CXT & SCM & TLD & ASLA & IVT & CPF & KCF & TGPR & DeepTrack \\
\hline tigerl & $0.17 / 0.13$ & 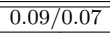 & $0.12 / 0.09$ & $0.37 / 0.17$ & $0.13 / 0.11$ & $0.46 / 0.36$ & $0.23 / 0.15$ & $\overline{00.08 / 0.07}$ & $\overline{00.39 / 0.24}$ & $0.97 / 0.94$ & $0.28 / 0.22$ & $0.56 / 0.36$ \\
\hline carDark & $1.00 / 1.00$ & $0.38 / 0.09$ & $0.74 / 0.66$ & $0.73 / 0.67$ & $1.00 / 0.98$ & $0.64 / 0.50$ & $1.00 / 0.99$ & $0.81 / 0.69$ & $0.17 / 0.02$ & $1.00 / 0.44$ & $1.00 / 0.95$ & $1.00 / 0.97$ \\
\hline girl & $1.00 / 0.90$ & $0.71 / 0.25$ & $0.95 / 0.41$ & $0.77 / 0.61$ & $1.00 / 0.74$ & $0.92 / 0.61$ & $1.00 / 0.78$ & $0.44 / 0.17$ & $0.74 / 0.40$ & $0.86 / 0.47$ & $0.92 / 0.69$ & $0.98 / 0.83$ \\
\hline david & $0.33 / 0.19$ & $0.70 / 0.05$ & $0.94 / 0.38$ & $1.00 / 0.48$ & $1.00 / 0.84$ & $1.00 / 0.83$ & $1.00 / 0.94$ & $1.00 / 0.65$ & $0.19 / 0.02$ & $1.00 / 0.26$ & $0.98 / 0.26$ & $1.00 / 0.76$ \\
\hline singerl & $0.64 / 0.20$ & $0.50 / 0.20$ & $1.00 / 0.36$ & $0.97 / 0.27$ & $1.00 / 1.00$ & $1.00 / 0.93$ & $1.00 / 0.98$ & $0.96 / 0.35$ & $0.99 / 0.10$ & $0.81 / 0.20$ & $0.68 / 0.19$ & $1.00 / 1.00$ \\
\hline skatingl & $0.47 / 0.20$ & $0.13 / 0.08$ & $0.90 / 0.43$ & $0.23 / 0.06$ & $0.77 / 0.21$ & $0.32 / 0.21$ & $0.77 / 0.45$ & $0.11 / 0.05$ & $0.23 / 0.17$ & $1.00 / 0.23$ & $0.81 / 0.25$ & $1.00 / 0.45$ \\
\hline deer & $1.00 / 0.94$ & $0.13 / 0.07$ & $0.04 / 0.03$ & $1.00 / 0.87$ & $0.03 / 0.03$ & $0.73 / 0.73$ & $0.03 / 0.03$ & $0.03 / 0.03$ & $0.04 / 0.03$ & $0.82 / 0.76$ & $0.86 / 0.79$ & $1.00 / 0.99$ \\
\hline singer 2 & $0.04 / 0.03$ & $0.40 / 0.27$ & $0.45 / 0.43$ & $0.06 / 0.04$ & $0.11 / 0.13$ & $0.07 / 0.05$ & $0.04 / 0.03$ & $0.04 / 0.04$ & $0.12 / 0.09$ & $0.95 / 0.89$ & $0.97 / 0.91$ & $0.57 / 0.34$ \\
\hline car4 & $0.99 / 0.26$ & $0.35 / 0.23$ & $0.36 / 0.32$ & $0.38 / 0.27$ & $0.97 / 0.93$ & $0.87 / 0.63$ & $1.00 / 0.95$ & $1.00 / 1.00$ & $0.14 / 0.01$ & $0.95 / 0.24$ & $1.00 / 0.28$ & $1.00 / 1.00$ \\
\hline tiger 2 & $0.63 / 0.42$ & $0.41 / 0.23$ & $0.16 / 0.08$ & $0.34 / 0.16$ & $0.11 / 0.05$ & $0.39 / 0.04$ & $0.14 / 0.11$ & $0.08 / 0.05$ & $0.11 / 0.04$ & $0.36 / 0.28$ & $0.72 / 0.47$ & $0.49 / 0.32$ \\
\hline dudek & $0.90 / 0.81$ & $0.69 / 0.76$ & $0.88 / 0.96$ & $0.82 / 0.87$ & $0.88 / 0.86$ & $0.60 / 0.63$ & $0.75 / 0.74$ & $0.89 / 0.88$ & $0.57 / 0.58$ & $0.88 / 0.82$ & $0.75 / 0.71$ & $0.73 / 0.81$ \\
\hline sylvester & $0.99 / 0.85$ & $0.65 / 0.46$ & $0.82 / 0.74$ & $0.85 / 0.56$ & $0.95 / 0.77$ & $0.95 / 0.80$ & $0.82 / 0.65$ & $0.68 / 0.63$ & $0.86 / 0.52$ & $0.84 / 0.73$ & $0.96 / 0.93$ & $1.00 / 0.92$ \\
\hline jumping & $1.00 / 0.50$ & $1.00 / 0.33$ & $0.21 / 0.08$ & $1.00 / 0.25$ & $0.15 / 0.11$ & $1.00 / 0.70$ & $0.45 / 0.15$ & $0.21 / 0.08$ & $0.16 / 0.09$ & $0.34 / 0.26$ & $0.95 / 0.50$ & $1.00 / 0.93$ \\
\hline david2 & $1.00 / 1.00$ & $0.98 / 0.24$ & $1.00 / 0.88$ & $1.00 / 1.00$ & $1.00 / 0.80$ & $1.00 / 0.70$ & $1.00 / 0.95$ & $1.00 / 0.74$ & $1.00 / 0.25$ & $1.00 / 1.00$ & $1.00 / 0.97$ & $1.00 / 0.87$ \\
\hline shaking & $0.19 / 0.04$ & $0.28 / 0.18$ & $0.93 / 0.83$ & $0.13 / 0.04$ & $0.81 / 0.69$ & $0.41 / 0.31$ & $0.48 / 0.17$ & $0.01 / 0.01$ & $0.17 / 0.07$ & $0.02 / 0.01$ & $0.97 / 0.70$ & $0.95 / 0.68$ \\
\hline trellis & $0.88 / 0.72$ & $0.23 / 0.16$ & $0.50 / 0.44$ & $0.97 / 0.69$ & $0.87 / 0.84$ & $0.53 / 0.45$ & $0.86 / 0.85$ & $0.33 / 0.26$ & $0.30 / 0.14$ & $1.00 / 0.74$ & $0.98 / 0.68$ & $1.00 / 0.96$ \\
\hline woman & $1.00 / 0.89$ & $0.21 / 0.18$ & $0.20 / 0.16$ & $0.37 / 0.15$ & $0.94 / 0.69$ & $0.19 / 0.15$ & $0.20 / 0.17$ & $0.20 / 0.17$ & $0.20 / 0.05$ & $0.94 / 0.90$ & $0.97 / 0.87$ & $0.98 / 0.24$ \\
\hline fish & $1.00 / 1.00$ & $0.39 / 0.28$ & $0.65 / 0.57$ & $1.00 / 1.00$ & $0.86 / 0.85$ & $1.00 / 0.96$ & $1.00 / 1.00$ & $1.00 / 1.00$ & $0.11 / 0.08$ & $1.00 / 1.00$ & $0.97 / 0.97$ & $1.00 / 1.00$ \\
\hline matrix & $0.12 / 0.12$ & $0.18 / 0.10$ & $0.22 / 0.03$ & $0.06 / 0.01$ & $0.35 / 0.24$ & $0.16 / 0.03$ & $0.05 / 0.01$ & $0.02 / 0.02$ & $0.09 / 0.02$ & $0.17 / 0.11$ & $0.39 / 0.26$ & $0.72 / 0.43$ \\
\hline ironman & $0.11 / 0.02$ & $0.11 / 0.02$ & $0.17 / 0.12$ & $0.04 / 0.03$ & $0.16 / 0.09$ & $0.12 / 0.04$ & $0.13 / 0.08$ & $0.05 / 0.05$ & $0.05 / 0.04$ & $0.22 / 0.10$ & $0.22 / 0.13$ & $0.08 / 0.05$ \\
\hline mhyang & $1.00 / 0.97$ & $0.46 / 0.25$ & $1.00 / 0.77$ & $1.00 / 1.00$ & $1.00 / 0.96$ & $0.98 / 0.52$ & $1.00 / 1.00$ & $1.00 / 1.00$ & $0.79 / 0.08$ & $1.00 / 0.93$ & $0.95 / 0.88$ & $1.00 / 0.96$ \\
\hline liquor & $0.39 / 0.40$ & $0.20 / 0.20$ & $0.52 / 0.52$ & $0.21 / 0.21$ & $0.28 / 0.29$ & $0.59 / 0.54$ & $0.23 / 0.23$ & $0.21 / 0.21$ & $0.52 / 0.53$ & $0.98 / 0.97$ & $0.27 / 0.27$ & $0.91 / 0.89$ \\
\hline motorRolling & $0.09 / 0.09$ & $0.04 / 0.06$ & $0.05 / 0.05$ & $0.04 / 0.02$ & $0.04 / 0.05$ & $0.12 / 0.10$ & $0.06 / 0.07$ & $0.03 / 0.04$ & $0.06 / 0.04$ & $0.05 / 0.05$ & $0.09 / 0.10$ & $0.80 / 0.43$ \\
\hline coke & $0.95 / 0.87$ & $0.15 / 0.08$ & $0.15 / 0.11$ & $0.65 / 0.15$ & $0.43 / 0.24$ & $0.68 / 0.09$ & $0.16 / 0.10$ & $0.13 / 0.13$ & $0.39 / 0.03$ & $0.84 / 0.41$ & $0.95 / 0.63$ & $0.91 / 0.18$ \\
\hline soccer & $0.25 / 0.15$ & $0.19 / 0.14$ & $0.45 / 0.18$ & $0.23 / 0.12$ & $0.27 / 0.16$ & $0.11 / 0.11$ & $0.12 / 0.11$ & $0.17 / 0.14$ & $0.26 / 0.16$ & $0.79 / 0.35$ & $0.16 / 0.13$ & $0.30 / 0.16$ \\
\hline boy & $1.00 / 0.93$ & $0.85 / 0.29$ & $0.97 / 0.61$ & $0.94 / 0.42$ & $0.44 / 0.44$ & $1.00 / 0.74$ & $0.44 / 0.44$ & $0.33 / 0.31$ & $1.00 / 0.82$ & $1.00 / 0.96$ & $0.99 / 0.91$ & $1.00 / 0.93$ \\
\hline basketball & $0.12 / 0.09$ & $0.28 / 0.20$ & $1.00 / 0.85$ & $0.04 / 0.02$ & $0.66 / 0.53$ & $0.03 / 0.02$ & $0.60 / 0.26$ & $0.50 / 0.08$ & $0.74 / 0.54$ & $0.92 / 0.71$ & $0.99 / 0.69$ & $0.82 / 0.39$ \\
\hline lemming & $0.63 / 0.49$ & $0.82 / 0.68$ & $0.51 / 0.42$ & $0.73 / 0.38$ & $0.17 / 0.16$ & $0.86 / 0.43$ & $0.17 / 0.17$ & $0.17 / 0.17$ & $0.88 / 0.40$ & $0.49 / 0.30$ & $0.35 / 0.26$ & $0.28 / 0.26$ \\
\hline bolt & $0.02 / 0.01$ & $0.01 / 0.01$ & $0.31 / 0.14$ & $0.03 / 0.01$ & $0.03 / 0.01$ & $0.31 / 0.08$ & $0.02 / 0.01$ & $0.01 / 0.01$ & $0.91 / 0.15$ & $0.99 / 0.75$ & $0.02 / 0.01$ & $0.99 / 0.78$ \\
\hline crossing & $1.00 / 0.72$ & $1.00 / 0.83$ & $0.44 / 0.36$ & $0.62 / 0.32$ & $1.00 / 0.99$ & $0.62 / 0.41$ & $1.00 / 0.99$ & $1.00 / 0.23$ & $0.89 / 0.38$ & $1.00 / 0.78$ & $1.00 / 0.81$ & $0.94 / 0.56$ \\
\hline couple & $0.74 / 0.51$ & $0.68 / 0.61$ & $0.11 / 0.06$ & $0.64 / 0.52$ & $0.11 / 0.11$ & $1.00 / 0.98$ & $0.09 / 0.09$ & $0.09 / 0.09$ & $0.87 / 0.58$ & $0.26 / 0.24$ & $0.60 / 0.35$ & $0.99 / 0.63$ \\
\hline david3 & $0.34 / 0.34$ & $0.74 / 0.60$ & $0.56 / 0.44$ & $0.15 / 0.10$ & $0.50 / 0.47$ & $0.11 / 0.10$ & $0.55 / 0.49$ & $0.75 / 0.41$ & $0.57 / 0.33$ & $1.00 / 0.96$ & $1.00 / 0.69$ & $1.00 / 0.93$ \\
\hline carScale & $0.65 / 0.37$ & $0.63 / 0.35$ & $0.55 / 0.42$ & $0.74 / 0.74$ & $0.65 / 0.64$ & $0.85 / 0.29$ & $0.74 / 0.65$ & $0.78 / 0.67$ & $0.67 / 0.32$ & $0.81 / 0.35$ & $0.79 / 0.37$ & $0.67 / 0.56$ \\
\hline doll & $0.92 / 0.34$ & $0.73 / 0.20$ & $0.97 / 0.73$ & $0.99 / 0.87$ & $0.98 / 0.97$ & $0.98 / 0.39$ & $0.92 / 0.91$ & $0.76 / 0.27$ & $0.94 / 0.84$ & $0.97 / 0.33$ & $0.94 / 0.40$ & $0.96 / 0.86$ \\
\hline skiing & $0.04 / 0.04$ & $0.07 / 0.06$ & $0.14 / 0.01$ & $0.15 / 0.06$ & $0.14 / 0.06$ & $0.12 / 0.05$ & $0.14 / 0.11$ & $0.11 / 0.09$ & $0.06 / 0.01$ & $0.07 / 0.05$ & $0.12 / 0.10$ & $0.09 / 0.06$ \\
\hline football & $0.75 / 0.57$ & $0.79 / 0.67$ & $0.80 / 0.65$ & $0.80 / 0.57$ & $0.77 / 0.42$ & $0.80 / 0.28$ & $0.73 / 0.62$ & $0.79 / 0.61$ & $0.97 / 0.60$ & $0.80 / 0.57$ & $1.00 / 0.75$ & $0.79 / 0.52$ \\
\hline football1 & $1.00 / 0.72$ & $1.00 / 0.55$ & $0.99 / 0.51$ & $1.00 / 0.96$ & $0.57 / 0.34$ & $0.55 / 0.34$ & $0.80 / 0.39$ & $0.81 / 0.49$ & $1.00 / 0.58$ & $0.96 / 0.80$ & $0.99 / 0.41$ & $1.00 / 0.38$ \\
\hline freemanl & $0.80 / 0.16$ & $0.94 / 0.12$ & $0.95 / 0.13$ & $0.73 / 0.18$ & $0.98 / 0.54$ & $0.54 / 0.18$ & $0.39 / 0.20$ & $0.81 / 0.26$ & $0.76 / 0.18$ & $0.39 / 0.13$ & $0.93 / 0.21$ & $1.00 / 0.35$ \\
\hline freeman3 & $0.79 / 0.12$ & $0.05 / 0.00$ & $0.72 / 0.22$ & $1.00 / 0.89$ & $1.00 / 0.88$ & $0.77 / 0.42$ & $1.00 / 0.90$ & $0.76 / 0.33$ & $0.17 / 0.14$ & $0.91 / 0.21$ & $0.77 / 0.15$ & $0.97 / 0.67$ \\
\hline freeman4 & $0.37 / 0.15$ & $0.20 / 0.02$ & $0.37 / 0.08$ & $0.43 / 0.17$ & $0.51 / 0.18$ & $0.41 / 0.24$ & $0.22 / 0.16$ & $0.35 / 0.17$ & $0.12 / 0.02$ & $0.53 / 0.12$ & $0.58 / 0.21$ & $0.71 / 0.22$ \\
\hline subway & $0.98 / 0.63$ & $0.99 / 0.68$ & $0.23 / 0.18$ & $0.26 / 0.20$ & $1.00 / 0.90$ & $0.25 / 0.22$ & $0.23 / 0.21$ & $0.22 / 0.19$ & $0.22 / 0.10$ & $1.00 / 0.94$ & $1.00 / 0.99$ & $1.00 / 0.79$ \\
\hline suv & $0.57 / 0.57$ & $0.12 / 0.12$ & $0.52 / 0.47$ & $0.91 / 0.90$ & $0.98 / 0.80$ & $0.91 / 0.70$ & $0.57 / 0.55$ & $0.45 / 0.44$ & $0.78 / 0.63$ & $0.98 / 0.98$ & $0.66 / 0.66$ & $0.52 / 0.52$ \\
\hline walking & $1.00 / 0.42$ & $1.00 / 0.37$ & $1.00 / 0.55$ & $0.24 / 0.22$ & $1.00 / 0.86$ & $0.96 / 0.30$ & $1.00 / 0.99$ & $1.00 / 0.98$ & $1.00 / 0.65$ & $1.00 / 0.34$ & $1.00 / 0.41$ & $1.00 / 0.94$ \\
\hline walking 2 & $0.98 / 0.32$ & $0.41 / 0.31$ & $0.41 / 0.39$ & $0.41 / 0.39$ & $1.00 / 0.99$ & $0.43 / 0.29$ & $0.40 / 0.40$ & $1.00 / 0.99$ & $0.36 / 0.35$ & $0.44 / 0.30$ & $0.99 / 0.31$ & $0.61 / 0.38$ \\
\hline mountainBike & $0.92 / 0.67$ & $0.67 / 0.41$ & $1.00 / 0.81$ & 0.28 & $0.97 / 0.72$ & $0.26 / 0.21$ & $0.90 / 0.82$ & $1.00 / 0.84$ & $0.15 / 0.06$ & $1.00 / 0.88$ & $1.00 / 0.87$ & $1.00 / 0.91$ \\
\hline faceoccl & $0.58 / 0.95$ & $0.22 / 0.46$ & $0.53 / 0.72$ & $0.34 / 0.57$ & $0.93 / 1.00$ & $0.20 / 0.65$ & $0.18 / 0.25$ & $0.64 / 0.87$ & $0.32 / 0.41$ & $0.73 / 0.99$ & $0.66 / 0.80$ & $0.33 / 0.42$ \\
\hline jogging-1 & $0.24 / 0.22$ & $0.23 / 0.21$ & $0.23 / 0.18$ & $0.96 / 0.95$ & $0.23 / 0.21$ & $0.97 / 0.95$ & $0.23 / 0.22$ & $0.22 / 0.22$ & $0.54 / 0.23$ & $0.23 / 0.22$ & $0.99 / 0.96$ & $0.97 / 0.94$ \\
\hline jogging-2 & $0.25 / 0.22$ & $0.19 / 0.16$ & $0.19 / 0.16$ & $0.16 / 0.15$ & $1.00 / 0.98$ & $0.86 / 0.83$ & $0.18 / 0.17$ & $0.20 / 0.19$ & $0.84 / 0.72$ & $0.16 / 0.15$ & $1.00 / 0.95$ & $0.99 / 0.30$ \\
\hline dog 1 & $1.00 / 0.51$ & $0.92 / 0.45$ & $0.83 / 0.61$ & $1.00 / 0.95$ & $0.98 / 0.76$ & $1.00 / 0.61$ & $1.00 / 0.87$ & $0.98 / 0.80$ & $0.91 / 0.90$ & $1.00 / 0.51$ & $1.00 / 0.52$ & $1.00 / 0.95$ \\
\hline fleetface & $0.64 / 0.51$ & $0.36 / 0.32$ & $0.66 / 0.68$ & $0.57 / 0.60$ & $0.53 / 0.58$ & $0.51 / 0.41$ & $0.30 / 0.32$ & $0.26 / 0.24$ & $0.16 / 0.21$ & $0.46 / 0.47$ & $0.45 / 0.47$ & $0.51 / 0.60$ \\
\hline faceocc 2 & $1.00 / 0.97$ & $0.74 / 0.62$ & $0.98 / 0.84$ & $1.00 / 0.90$ & $0.86 / 0.74$ & $0.86 / 0.51$ & $0.79 / 0.61$ & $0.99 / 0.77$ & $0.40 / 0.29$ & $0.97 / 0.79$ & $0.47 / 0.45$ & $1.00 / 0.71$ \\
\hline Overall & $0.66 / 0.48$ & $0.47 / 0.28$ & $0.58 / 0.41$ & $0.58 / 0.43$ & $0.65 / 0.55$ & $0.61 / 0.42$ & $0.53 / 0.46$ & $0.50 / 0.38$ & $0.49 / 0.28$ & $0.74 / 0.53$ & $0.77 / 0.54$ & $0.83 / 0.63$ \\
\hline No. Best & 21 & 4 & 10 & 16 & 19 & 11 & 18 & 11 & 4 & 28 & 21 & 33 \\
\hline No. Bad & 62 & 89 & 71 & 66 & 51 & 72 & 64 & 74 & 84 & 48 & 45 & 36 \\
\hline
\end{tabular}

TABLE I

THE TRACKING SCORES OF DEEPTRACK AND OTHER VISUAL TRACKERS ON THE CVPR2013 BENCHMARK. THE REPORTED RESULTS ARE SHOWN IN THE ORDER OF "TP/TSR". THE TOP SCORES ARE SHOWN IN RED FOR EACH ROW. A SCORE IS SHOWN IN BLUE IF IT IS HIGHER THAN $80 \%$ OF THE HIGHEST VALUE IN THAT ROW. "NO. BEST" ROW SHOWS THE NUMBER OF BEST SCORES FOR EACH TRACKING ALGORITHM WHILE "NO. BAD" ROW SHOWS THE NUMBER OF LOW SCORES, $i$.E., THE SCORES LOWER THAN $80 \%$ OF THE MAXIMUM ONE IN THE CORRESPONDING ROW.

initializing it on the ground truth bounding box. The setting of Experiment-2 is the same to Experiment-1, except that the initial bounding box is randomly perturbed in the order of ten percent of the object size. In Experiment-3, the colorful frames are converted into grayscale images.

Firstly, we follow the evaluation protocol to test our method, compared with other 27 tracking algorithms provided in the benchmark website. The main comparison results can be found in Table $\mathrm{II}$ and Fig. 7. We can see that, in average, the proposed method ranks the first for both accuracy and robustness comparison. In specific, DeepTrack achieves the best robustness scores for all the scenarios while ranks the second in accuracy for all the experimental settings. In the Fig. 7. one can observe that the red circles (which stands for DeepTrack) always locate in the top-right corner of the plot. This observation is consistent to the scores reported in Table [I] From the result we can see that our DeepTrack achieves close while consistently better performances than the PLT method [27]. Other tracking methods that can achieve similar performances on this benchmarks are FoT [36], EDFT [37] and LGT++ [38].

Note that the scores listed in Table II and the plots in Fig. 7 are rank-based, which is different from the measuring criterion used in the CVPR2013 benchmark. It is well-known that the evaluation method for visual tracker is not unique and could be sophisticated for a specific objective [39]. Usually different tracker measures offer different points of view for accessing the tracking method. The best performance on the VOT2013 benchmark justifies the superiority of DeepTrack, from another perspective.

In [28], the authors perform their TGPR tracker on the VOT2013 benchmark, without comparing with other trackers. We here compare our DeepTrack with the TGPR algorithm, which is recently proposed and achieves state-of-the-art performance in the CVPR2013 benchmark. Following the settings in [28], we perform the proposed tracker in Experiment-1 and Experiment-2. The performance comparison is shown in Table III. 

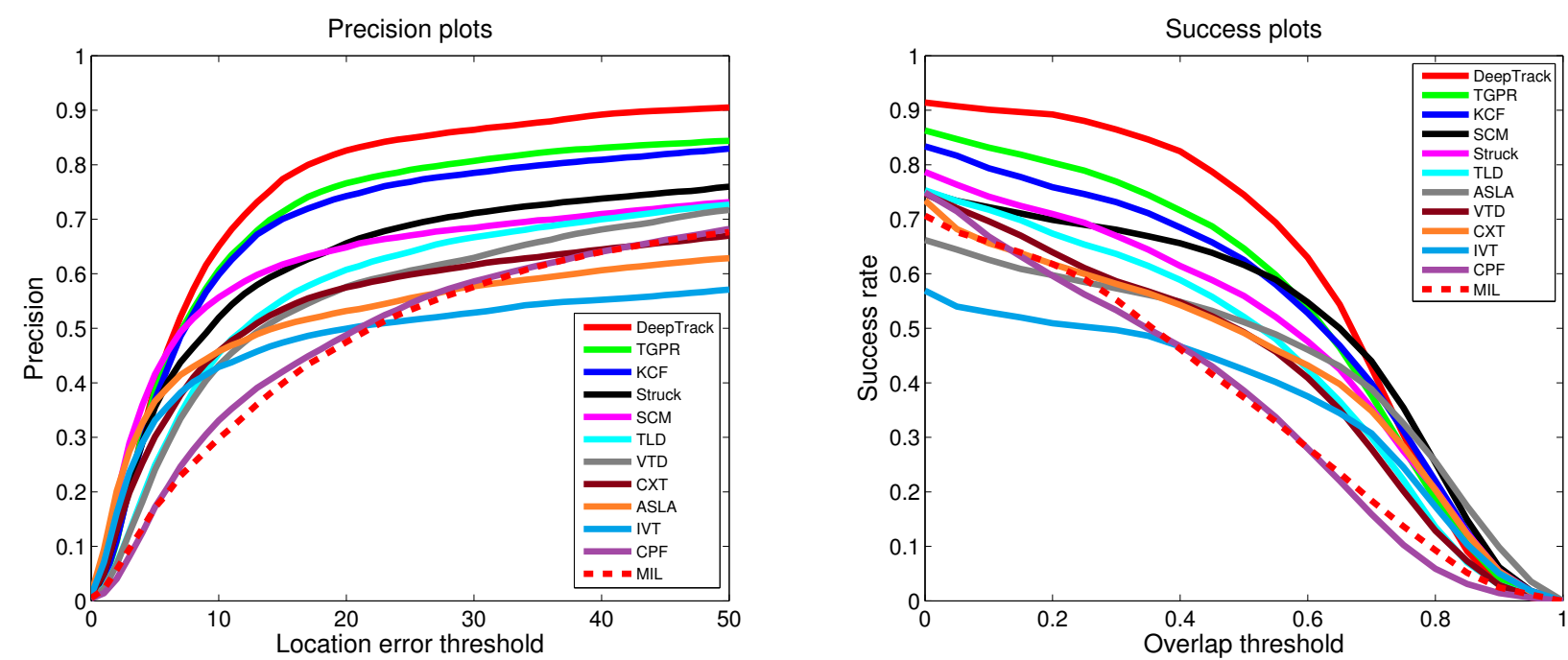

Fig. 5. The Precision Plot (left) and the Success Plot (right) of the tracking results on the CVPR2013 benchmark. Note that the color of one curve is determined by the rank of the corresponding trackers, not their names.

\begin{tabular}{|c|c|c|c|c|c|c|c|c|c|c|c|c|c|c|c|c|c|}
\hline & bicycle & "bolt & car & cup & david & diving & face & gym & hand & iceskater & $\overline{\text { juice }}$ & $\overline{\text { jump }}$ & singer & sunshade & torus & woman & " overall \\
\hline "Exp1-TPGR-Rob. & $\overline{0}$ & 1.27 & 0.40 & 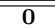 & $\begin{array}{ll}0.27 \\
\end{array}$ & 2.87 & 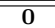 & 2.87 & $\begin{array}{ll}1.67 \\
\end{array}$ & 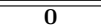 & $\overline{0}$ & $\overline{0}$ & 0.60 & 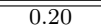 & "0.13 & 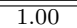 & "0.71 \\
\hline Exp1-DeepTrack-Rob. & 0.47 & 0.07 & 0.47 & 0 & 0.20 & 0.80 & 0 & 0.73 & 0.20 & 0 & $\mathbf{0}$ & $\mathbf{0}$ & 0 & $\mathbf{0}$ & 0.07 & 0.47 & 0.22 \\
\hline Exp1-TPGR-Accu. & 0.60 & 0.57 & 0.45 & 0.83 & 0.58 & 0.33 & 0.85 & 0.57 & 0.56 & 0.60 & 0.76 & 0.59 & 0.65 & 0.73 & 0.78 & 0.74 & 0.64 \\
\hline Exp1-DeepTrack-Accu. & 0.58 & 0.61 & 0.51 & 0.86 & 0.54 & 0.35 & 0.73 & 0.49 & 0.54 & 0.61 & 0.81 & 0.66 & 0.51 & 0.72 & 0.76 & 0.60 & 0.62 \\
\hline Exp2-TPGR-Rob. & $\overline{0}$ & 1.27 & 0.20 & $\overline{0}$ & 0.27 & 2.87 & 0.07 & 3.00 & 2.07 & $\overline{0}$ & $\overline{0}$ & $\overline{0}$ & 0.33 & 0.07 & 0.60 & 1.00 & 0.73 \\
\hline Exp2-DeepTrack-Rob. & 0.27 & $\mathbf{0}$ & 0.33 & 0 & 0.20 & 0.80 & 0 & 0.27 & 0.60 & $\mathbf{0}$ & $\mathbf{0}$ & $\mathbf{0}$ & 0 & 0.07 & 0.27 & 0.67 & 0.22 \\
\hline Exp2-TPGR-Accu. & 0.57 & 0.57 & 0.41 & 0.75 & 0.58 & 0.32 & 0.77 & 0.53 & 0.53 & 0.57 & 0.73 & 0.57 & 0.45 & 0.64 & 0.65 & 0.67 & 0.58 \\
\hline Exp2-DeepTrack-Accu. & 0.54 & 0.62 & 0.49 & 0.77 & 0.50 & 0.36 & 0.70 & 0.47 & 0.53 & 0.59 & 0.75 & 0.62 & 0.60 & 0.69 & 0.69 & 0.56 & 0.59 \\
\hline
\end{tabular}

TABLE III

THE PERFORMANCE COMPARISON BETWEen DEEPTRACK TRACKER AND THE TPGR TRACKER ON THE VOT2013 BENCHMARK. THE BETTER ROBUSTNESS SCORE IS SHOWN IN BOLD. NOTE THAT FOR ACCURACY (ACCU.), THE COMPARISON IS NOT FAIR IF THE ROBUSTNESS SCORE IS DIFFERENT AND THUS NO BOLD ACCURACY SCORE IS SHOWN.

We can see that the proposed DeepTrack outperforms the TPGR tracker in the robustness evaluation, with a clear performance gap. For Experiment-1, one needs to reinitialize the TPGR tracker for 0.71 times per sequence while that number for our method is only 0.22 . Similarly, with the bounding box perturbation (Experiment-2), TPGR needs 0.73 times reinitialization while DeepTrack still requires 0.22 times. Note that in Table III the accuracies from different trackers are not directly comparable, as they are calculated based on different re-initialization conditions. However, by observing the overall scores, we can still draw the conclusion that the DeepTrack is more robust than TPGR as it achieves similar accuracies to TPGR (0.62 v.s. 0.64 for Experiment- 1 and 0.59 v.s. 0.58 for Experiment-2) while only requires around one third of reinitializations.

\section{Verification for the structural loss and the robust temporal sampling}

Here we verify the three proposed modifications to the CNN model. We rerun the experiment on the CVPR2013 benchmark using the DeepTrack with each modification inactivated. In specific, the temporal sampling mechanism, the label uncertainty and the structural loss is disabled and the yielded tracking results are shown in Fig. 8, compared with the full-version of the proposed method. Beside, the results of two state-of-the-art method, $i$.e., Struck and TPGR are also shown as references.

From the figure we can see that, the structural loss, the temporal sampling mechanism and the label uncertainty all contribute the success of our CNN tracker. In particular, the temporal sampling plays a more important role. The structural loss can increase the TP accuracy by $10 \%$ and one can lifts the TP accuracy by $4 \%$ when the label noise is taken into consideration. Generally speaking, the curve consistently goes down when one component are removed from the original DeepTrack model. That indicates the validity of the propose modifications.

\section{E. Tracking speed analysis}

We report the average speed (in fps) of the proposed DeepTrack method in Table IV, compared with the DeepTrack without the truncated loss. Note that there are two kinds of average speed scores: the average fps over all the sequences and the average fps over all the frames. The latter one reduces the influence of short sequences where the initialization process usually dominates the computational burden.

According to the table, the truncated loss boosts the tracking efficiency by around $37 \%$. Furthermore, our method tracks the object at an average speed around 2.5fps. Considering that the speed of TPGR is around 3fps [28] and for the Sparse 

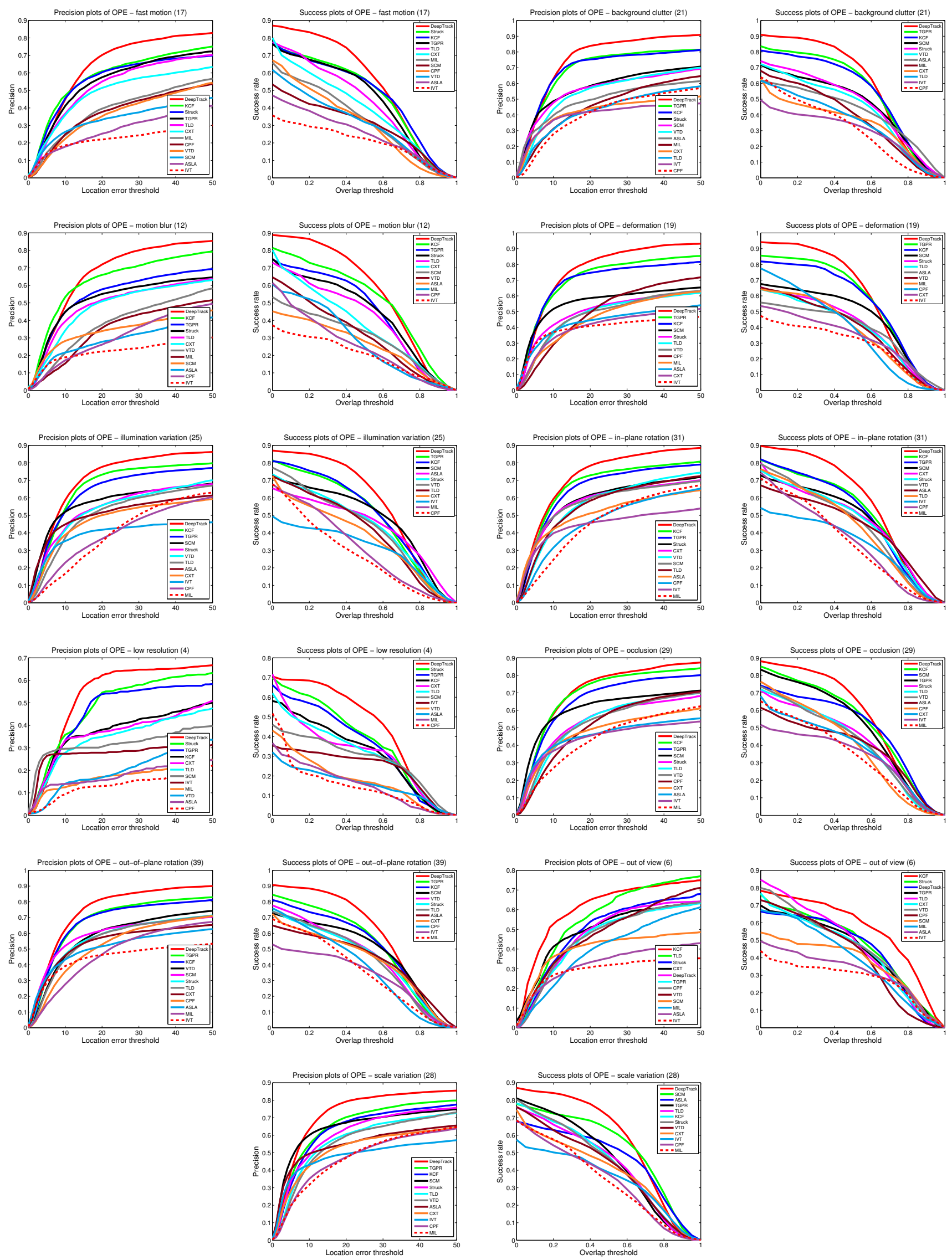

Fig. 6. The Precision Plot (left) and the Success Plot (right) of the tracking results on the CVPR2013 benchmark, for 11 kinds of tracking difficulties. 

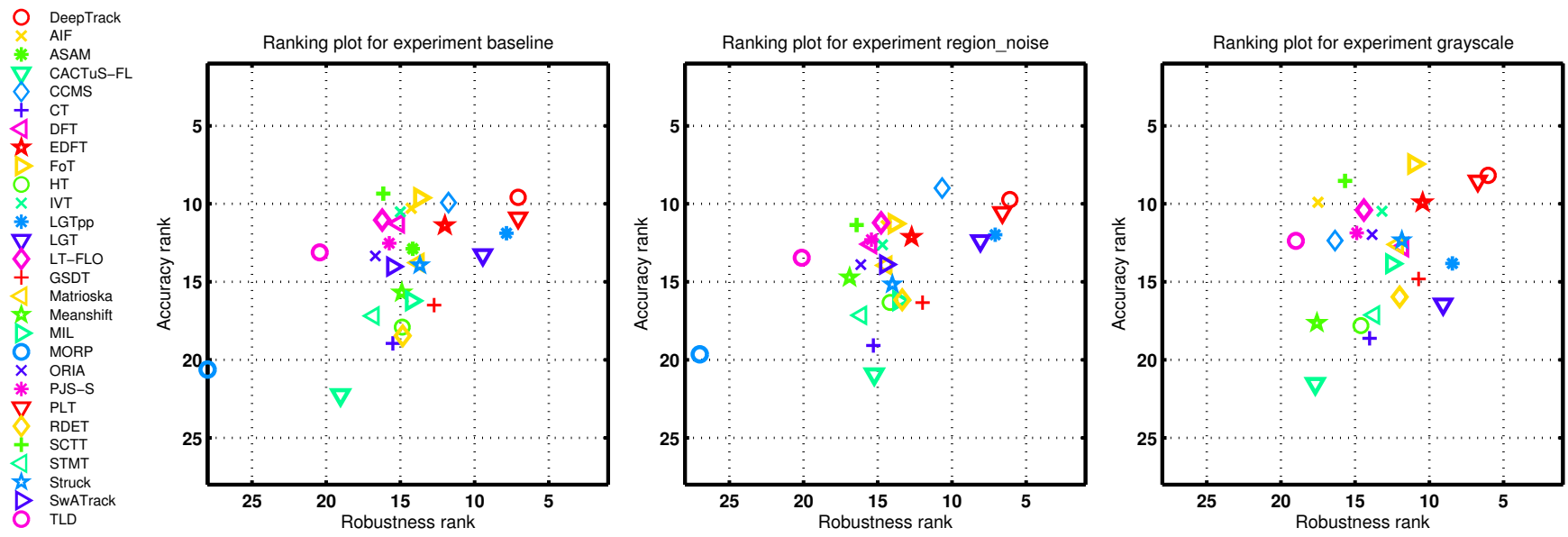

Fig. 7. The Precision Plot (left) and the Success Plot (right). The color of one curve is determined by the rank of the corresponding trackers, not their names.
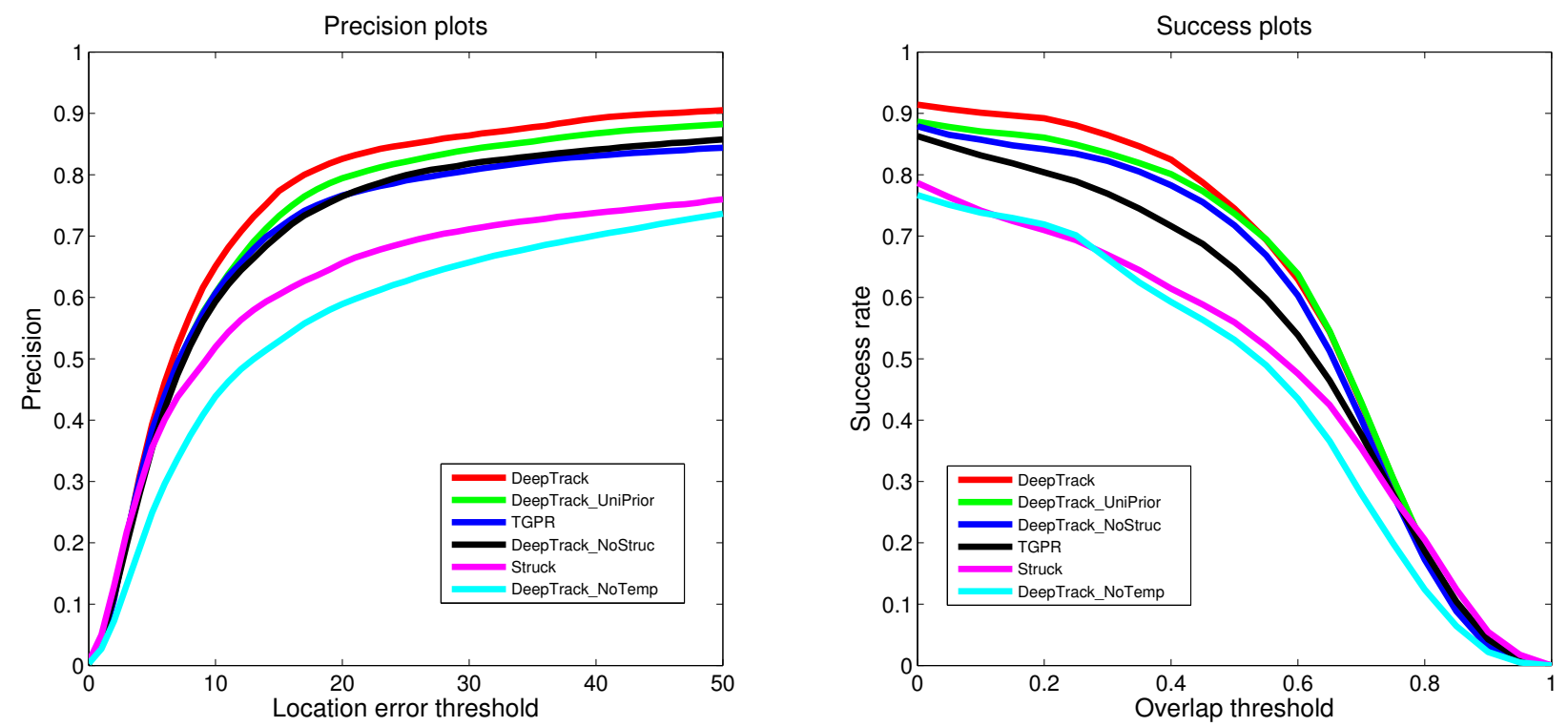

Fig. 8. The Precision Plot (left) and the Success Plot (right) of the results obtained by using different versions of DeepTrack. Note that the color of one curve is determined by the rank of the corresponding trackers, not their names.

Representation based methods the speeds are usually lower than 2.5fps [23]. We thus can draw the conclusion that the DeepTrack can achieve comparable speed to the state-of-theart methods.

\section{CONCLUSION}

We introduced a CNN based online object tracker. We employed a novel CNN architecture and a structural loss function that handles multiple input cues. We also proposed to modify the ordinary Stochastic Gradient Descent for visual tracking by iteratively update the parameters and add a robust temporal sampling mechanism in the mini-batch generation. This tracking-tailored SGD algorithm increase the speed and the robustness of the training process significantly. Our experiments demonstrated that the CNN-based DeepTrack outperforms state-of-the-art methods on two recently proposed benchmarks which contain over 60 video sequences and achieves the comparable tracking speed.

\section{REFERENCES}

[1] Patrick Pérez, Carine Hue, Jaco Vermaak, and Michel Gangnet, "Colorbased probabilistic tracking," in ECCV 2002.

[2] Robert T. Collins, Yanxi Liu, and Marius Leordeanu, "Online selection of discriminative tracking features," IEEE Transactions on Pattern Analysis and Machine Intelligence, vol. 27, no. 10, pp. 1631-1643, 2005.

[3] Amit Adam, Ehud Rivlin, and Ilan Shimshoni, "Robust fragments-based tracking using the integral histogram," in CVPR 2006, vol. 1.

[4] Sam Hare, Amir Saffari, and Philip HS Torr, "Struck: Structured output tracking with kernels," in ICCV 2011. IEEE, pp. 263-270.

[5] David G Lowe, "Distinctive image features from scale-invariant keypoints," International journal of computer vision, vol. 60, no. 2, pp. 91-110, 2004

[6] Navneet Dalal and Bill Triggs, "Histograms of oriented gradients for human detection," in Computer Vision and Pattern Recognition, 2005. CVPR 2005. IEEE Computer Society Conference on. IEEE, 2005, vol. 1, pp. 886-893. 


\begin{tabular}{l|c|c|c|c|c|c|c|c}
\hline \hline & \multicolumn{2}{|c|}{ Experiment-1 } & \multicolumn{2}{c|}{ Experiment-2 } & \multicolumn{2}{c|}{ Experiment-3 } & \multicolumn{2}{c}{ Averaged } \\
\cline { 2 - 9 } & Accu. & Rob. & Accu. & Rob. & Accu. & Rob. & Accu. & Rob. \\
\hline CNN & 9.60 & 7.06 & 10.14 & 6.09 & 8.17 & 6.04 & 9.30 & 6.40 \\
AIF & 10.29 & 14.27 & 11.39 & 14.43 & 9.90 & 17.50 & 10.52 & 15.40 \\
ASAM & 12.86 & 14.17 & NaN & NaN & NaN & NaN & NaN & NaN \\
CACTuS-FL & 22.27 & 19.03 & 20.92 & 15.24 & 21.54 & 17.69 & 21.58 & 17.32 \\
CCMS & 9.87 & 11.76 & 8.97 & 10.66 & 12.36 & 16.35 & 10.40 & 12.92 \\
CT & 18.92 & 15.51 & 19.10 & 15.30 & 18.62 & 14.03 & 18.88 & 14.95 \\
DFT & 11.23 & 15.12 & 12.64 & 15.47 & 12.78 & 11.79 & 12.21 & 14.13 \\
EDFT & 11.45 & 12.00 & 12.18 & 12.72 & 9.91 & 10.44 & 11.18 & 11.72 \\
FoT & 9.63 & 13.69 & 11.23 & 13.87 & 7.44 & 11.04 & 9.44 & 12.87 \\
HT & 17.90 & 14.88 & 16.29 & 14.17 & 17.81 & 14.61 & 17.33 & 14.55 \\
IVT & 10.51 & 15.00 & 12.66 & 14.68 & 10.48 & 13.19 & 11.21 & 14.29 \\
LGTpp & 11.89 & 7.84 & 11.98 & 7.08 & 13.82 & 8.44 & 12.56 & 7.79 \\
LGT & 13.27 & 9.44 & 12.37 & 8.08 & 16.43 & 9.07 & 14.02 & 8.86 \\
LT-FLO & 11.09 & 16.22 & 11.25 & 14.77 & 10.40 & 14.41 & 10.91 & 15.14 \\
GSDT & 16.51 & 12.73 & 16.36 & 11.98 & 14.82 & 10.73 & 15.90 & 11.81 \\
Matrioska & 13.77 & 13.78 & 13.94 & 14.43 & 12.60 & 12.13 & 13.44 & 13.45 \\
Meanshift & 15.69 & 14.91 & 14.82 & 16.90 & 17.64 & 17.57 & 16.05 & 16.46 \\
MIL & 16.38 & 14.25 & 16.28 & 13.58 & 13.84 & 12.54 & 15.50 & 13.46 \\
MORP & 20.64 & 28.00 & 19.65 & 27.00 & NaN & NaN & NaN & NaN \\
ORIA & 13.13 & 16.69 & 13.86 & 16.15 & 11.97 & 13.85 & 12.99 & 15.56 \\
PJS-S & 12.50 & 15.75 & 12.31 & 15.43 & 11.87 & 14.89 & 12.22 & 15.36 \\
PLT & 10.88 & 7.06 & 10.58 & 6.60 & 8.54 & 6.73 & 10.00 & 6.79 \\
RDET & 18.42 & 14.84 & 16.14 & 13.35 & 15.97 & 12.00 & 16.84 & 13.40 \\
SCTT & 9.36 & 16.16 & 11.37 & 16.43 & 8.53 & 15.68 & 9.75 & 16.09 \\
STMT & 17.16 & 16.81 & 17.17 & 16.12 & 17.12 & 13.73 & 17.15 & 15.55 \\
Struck & 13.92 & 13.69 & 15.21 & 14.02 & 12.33 & 11.85 & 13.82 & 13.19 \\
SwATrack & 13.98 & 15.53 & 13.93 & 14.48 & NaN & NaN & NaN & NaN \\
TLD & 13.12 & 20.44 & 13.37 & 20.12 & 12.37 & 19.00 & 12.95 & 19.85 \\
\hline \hline
\end{tabular}

TABLE II

THE PERFORMANCE COMPARISON BETWEEN CNN TRACKER AND OTHER 27 TRACKERS ON THE VOT2013 BENCHMARK. FOR EACH COLUMN, THE BEST SCORE IS SHOWN IN BOLD AND RED WHILE THE SECOND BEST SCORE IS SHOWN IN BLUE.

\begin{tabular}{|c|c|c|}
\hline & Sequence Average & Frame Average \\
\hline With TruncLoss & $1.96 \mathrm{fps}$ & $2.52 \mathrm{fps}$ \\
\hline No TruncLoss & $1.49 \mathrm{fps}$ & $1.86 \mathrm{fps}$ \\
\hline
\end{tabular}

TABLE IV

THE TRACKING SPEED OF DEEPTRACK WITH OR WITHOUT THE TRUNCATED LOSS. NOTE THAT THERE ARE TWO KINDS OF KINDS OF AVERAGE SPEED SCORES: THE AVERAGE FPS OVER ALL THE SEQUENCES (SEQUENCE AVERAGE) AND THE AVERAGE FPS OVER ALl THE FRAMES (Frame Average).

[7] Timo Ahonen, Abdenour Hadid, and Matti Pietikainen, "Face description with local binary patterns: Application to face recognition," Pattern Analysis and Machine Intelligence, IEEE Transactions on, vol. 28, no. 12, pp. 2037-2041, 2006

[8] Y. Bengio, A. Courville, and P. Vincent, "Representation learning: A review and new perspectives," Pattern Analysis and Machine Intelligence, IEEE Transactions on, vol. 35, no. 8, pp. 1798-1828, 2013.

[9] Koray Kavukcuoglu, Pierre Sermanet, Y-Lan Boureau, Karol Gregor, Michaël Mathieu, and Yann LeCun, "Learning convolutional feature hierachies for visual recognition," in NIPS 2010.

[10] Alex Krizhevsky, Ilya Sutskever, and Geoffrey Hinton, "Imagenet classification with deep convolutional neural networks," in NIPS 2012

[11] Dan Claudiu Ciresan, Ueli Meier, and Jürgen Schmidhuber, "Multicolumn deep neural networks for image classification," in CVPR 2012.

[12] Ross Girshick, Jeff Donahue, Trevor Darrell, and Jitendra Malik, "Rich feature hierarchies for accurate object detection and semantic segmentation," in Computer Vision and Pattern Recognition (CVPR), 2014 IEEE Conference on. IEEE, 2014, pp. 580-587.

[13] Ning Zhang, Jeff Donahue, Ross Girshick, and Trevor Darrell, "Partbased r-cnns for fine-grained category detection," in Computer VisionECCV 2014, pp. 834-849. Springer, 2014.

[14] Jialue Fan, Wei Xu, Ying Wu, and Yihong Gong, "Human tracking using convolutional neural networks," Trans. Neur. Netw., vol. 21, no. 10, pp. 1610-1623, Oct. 2010.

[15] Naiyan Wang and Dit-Yan Yeung, "Learning a deep compact image representation for visual tracking," in NIPS 2013.

[16] Boris Babenko, Ming-Hsuan Yang, and Serge Belongie, "Visual tracking with online multiple instance learning," in Computer Vision and Pattern Recognition, 2009. CVPR 2009. IEEE Conference on. IEEE, 2009, pp. 983-990.
[17] Neil D Lawrence and Bernhard Schölkopf, "Estimating a kernel fisher discriminant in the presence of label noise," in ICML. Citeseer, 2001, pp. 306-313.

[18] Philip M Long and Rocco A Servedio, "Random classification noise defeats all convex potential boosters," Machine Learning, vol. 78, no. 3, pp. 287-304, 2010.

[19] Nagarajan Natarajan, Inderjit S Dhillon, Pradeep K Ravikumar, and Ambuj Tewari, "Learning with noisy labels," in Advances in Neural Information Processing Systems, 2013, pp. 1196-1204.

[20] Hanxi Li, Yi Li, and Fatih Porikli, "Deeptrack: Learning discriminative feature representations by convolutional neural networks for visual tracking," BMVC2014, 2014.

[21] Hanxi Li, Yi Li, and Fatih Porikli, "Robust online visual tracking with an single convolutional neural network," ACCV2014, 2014.

[22] Mark Everingham, Luc Van Gool, Christopher KI Williams, John Winn, and Andrew Zisserman, "The pascal visual object classes (voc) challenge," Intl J. of Comp. Vis., vol. 88, no. 2, pp. 303-338, 2010.

[23] Junliang Xing, Jin Gao, Bing $\mathrm{Li}$, Weiming $\mathrm{Hu}$, and Shuicheng Yan, "Robust object tracking with online multi-lifespan dictionary learning," in Computer Vision (ICCV), 2013 IEEE International Conference on. IEEE, 2013, pp. 665-672.

[24] Boris Babenko, Ming-Hsuan Yang, and Serge Belongie, "Visual tracking with online multiple instance learning," Transactions on Pattern Analysis and Machine Intelligence, August 2011.

[25] Paul Viola, John Platt, Cha Zhang, et al., "Multiple instance boosting for object detection," in NIPS, 2005, vol. 2, p. 5.

[26] Yi Wu, Jongwoo Lim, and Ming-Hsuan Yang, "Online object tracking: A benchmark," CVPR 2013.

[27] Matej Kristan, Roman Pflugfelder, Ale Leonardis, Jiri Matas, Fatih Porikli, Luka Cehovin, Georg Nebehay, Gustavo Fernandez, Toma Vojir, Adam Gatt, et al., "The visual object tracking vot2013 challenge results," in Computer Vision Workshops (ICCVW), 2013 IEEE International Conference on. IEEE, 2013, pp. 98-111.

[28] Jin Gao, Haibin Ling, Weiming Hu, and Junliang Xing, "Transfer learning based visual tracking with gaussian processes regression," in Computer Vision-ECCV 2014, pp. 188-203. Springer, 2014.

[29] J. F. Henriques, R. Caseiro, P. Martins, and J. Batista, "High-speed tracking with kernelized correlation filters," Pattern Analysis and Machine Intelligence, IEEE Transactions on, 2015.

[30] Zdenek Kalal, Jiri Matas, and Krystian Mikolajczyk, "Pn learning: Bootstrapping binary classifiers by structural constraints," in CVPR 2010. IEEE, pp. 49-56.

[31] Junseok Kwon and Kyoung Mu Lee, "Visual tracking decomposition," in in CVPR, 2010.

[32] Thang Ba Dinh, Nam Vo, and Gérard Medioni, "Context tracker: Exploring supporters and distracters in unconstrained environments," in CVPR 2011. IEEE, pp. 1177-1184.

[33] Xu Jia, Huchuan Lu, and Ming-Hsuan Yang, "Visual tracking via adaptive structural local sparse appearance model," in CVPR 2012. IEEE, pp. 1822-1829.

[34] Wei Zhong, Huchuan Lu, and Ming-Hsuan Yang, "Robust object tracking via sparsity-based collaborative model," in CVPR 2012. IEEE, pp. $1838-1845$

[35] David A. Ross, Jongwoo Lim, Ruei-Sung Lin, and Ming-Hsuan Yang, "Incremental learning for robust visual tracking," Intl. J. Comp. Vis, vol. 77 , no. 1-3, pp. 125-141, May 2008.

[36] Andreas Wendel, Sabine Sternig, and Martin Godec, "Robustifying the flock of trackers," in 16th Computer Vision Winter Workshop. Citeseer, 2011, p. 91.

[37] Michael Felsberg, "Enhanced distribution field tracking using channel representations," in Computer Vision Workshops (ICCVW), 2013 IEEE International Conference on. IEEE, 2013, pp. 121-128.

[38] Jingjing Xiao, Rustam Stolkin, and Ale Leonardis, "An enhanced adaptive coupled-layer lgtracker++," in Computer Vision Workshops (ICCVW), 2013 IEEE International Conference on. IEEE, 2013, pp. 137-144.

[39] Tahir Nawaz and Andrea Cavallaro, "A protocol for evaluating video trackers under real-world conditions," Image Processing, IEEE Transactions on, vol. 22, no. 4, pp. 1354-1361, 2013. 